\title{
Spectroscopic and photometric oscillatory envelope variability during the S Doradus outburst of the luminous blue variable R71
}

\author{
A. Mehner ${ }^{1}$, D. Baade ${ }^{2}$, J. H. Groh ${ }^{3}$, T. Rivinius ${ }^{1}$, F.-J. Hambsch ${ }^{4}, 5$, E. S. Bartlett ${ }^{1}$, D. Asmus ${ }^{1}$, \\ C. Agliozzo ${ }^{6,7}$, T. Szeifert ${ }^{1}$, and O. Stahl $^{8}$ \\ ${ }^{1}$ ESO - European Organisation for Astronomical Research in the Southern Hemisphere, Alonso de Cordova 3107, Vitacura, \\ Santiago de Chile, Chile \\ e-mail: amehner@eso.org \\ 2 ESO - European Organisation for Astronomical Research in the Southern Hemisphere, Karl-Schwarzschild-Straße 2, \\ 85748 Garching, Germany \\ 3 School of Physics, Trinity College Dublin, Dublin 2, Ireland \\ 4 AAVSO - American Association of Variable Star Observers, Cambridge, MA 02138, USA \\ 5 ROAD - Remote Observatory Atacama Desert, Vereniging Voor Sterrenkunde (VVS), Oude Bleken 12, 2400 Mol, Belgium \\ ${ }^{6}$ Millennium Institute of Astrophysics (MAS), Nuncio Monsenor Sótero Sanz 100, Providencia, 75000 Santiago, Chile \\ 7 Departamento de Ciencias Fisicas, Universidad Andres Bello, Avda. Republica 252, Santiago 8320000, Chile \\ 8 Zentrum für Astronomie der Universität Heidelberg, Landessternwarte, Königstuhl 12, 69117 Heidelberg, Germany
}

Received 25 August 2017 / Accepted 19 September 2017

\begin{abstract}
Context. Luminous blue variables (LBVs) are evolved massive stars that exhibit instabilities that are not yet understood. Stars can lose several solar masses during this evolutionary phase. The LBV phenomenon is thus critical to our understanding of the evolution of the most massive stars.

Aims. The LBV R71 in the Large Magellanic Cloud is presently undergoing an S Doradus outburst, which started in 2005. To better understand the LBV phenomenon, we determine the fundamental stellar parameters of R71 during its quiescence phase. In addition, we analyze multiwavelength spectra and photometry obtained during the current outburst.

Methods. We analyzed pre-outburst CASPEC spectra from 1984-1997, EMMI spectra in 2000, UVES spectra in 2002, and FEROS spectra from 2005 with the radiative transfer code CMFGEN to determine the fundamental stellar parameters of the star. A spectroscopic monitoring program with VLT X-shooter since 2012 secured visual to near-infrared spectra throughout the current outburst, which is well-covered by ASAS and AAVSO photometry. Mid-infrared images and radio data were also obtained.

Results. During quiescence, R71 has an effective temperature of $T_{\text {eff }}=15500 \mathrm{~K}$ and a luminosity of $\log \left(L_{*} / L_{\odot}\right)=5.78$. We determine its mass-loss rate to $4.0 \times 10^{-6} M_{\odot} \mathrm{yr}^{-1}$. We present the spectral energy distribution of R71 from the near-ultraviolet to the mid-infrared during its present outburst. Semi-regular oscillatory variability in the light curve of the star is observed during the current outburst. Absorption lines develop a second blue component on a timescale of twice that length. The variability may consist of one (quasi-)periodic component with $P \sim 425 / 850 \mathrm{~d}$ with additional variations superimposed.

Conclusions. R71 is a classical LBV, but this star is at the lower luminosity end of this group. Mid-infrared observations suggest that we are witnessing dust formation and grain evolution. During its current S Doradus outburst, R71 occupies a region in the HR diagram at the high-luminosity extension of the Cepheid instability strip and exhibits similar irregular variations as RV Tau variables. LBVs do not pass the Cepheid instability strip because of core evolution, but they develop comparable cool, low-mass, extended atmospheres in which convective instabilities may occur. As in the case of RV Tau variables, the occurrence of double absorption lines with an apparent regular cycle may be due to shocks within the atmosphere and period doubling may explain the factor of two in the lengths of the photometric and spectroscopic cycles.
\end{abstract}

Key words. stars: massive - stars: variables: S Doradus - stars: mass-loss - stars: individual: R71

\section{Introduction}

Luminous blue variables (LBVs), also known as S Doradus variables, are evolved massive stars that exhibit instabilities that are not yet understood (Conti 1984, 1997; Humphreys \& Davidson 1994; Nota \& Lamers 1997, and references therein). They represent a brief but critical phase in massive star evolution because several solar masses can be expelled during this stage

\footnotetext{
* Based on observations collected at ESO's Very Large Telescope under Prog-IDs: 69.D-0390(D), 289.D-5040(A), 290.D5032(A), 091.D-0116(A, B), 092.D-0024(A), 094.D-0266(A, B, C), 096.D-0043(A, B, C), 097.D-0006(A, B), 598.D-0005(A, B) and at the MPG/ESO 2.2-m Telescope under Prog-IDs: 076.D-0609(A), 078.D-0790(B), 086.D-0997(A, B), 087.D-0946(A), 089.D-0975(A), 094.A-9029(D), 096.A-9039(A), 097.D-0612(A, B), 098.D-0071(A).
}

(see, e.g., Groh et al. 2014). The LBV phenomenon extends to luminosities as low as $\log \left(L / L_{\odot}\right) \sim 5.2$, corresponding to stars with initial masses of $\sim 20 M_{\odot}$. Luminous blue variables experience outbursts with enhanced mass loss during which they appear to make transitions in the Hertzsprung-Russell (HR) diagram from their quiescent hot state $\left(T_{\text {eff }} \sim 16000-30000 \mathrm{~K}\right)$ to lower temperatures $\left(T_{\text {eff }} \sim 8000 \mathrm{~K}\right)$. Their instability could be responsible for the empirically found upper luminosity boundary above and to the right of which no supergiants are found (Humphreys \& Davidson 1979, 1984). The lowest luminosity LBVs may have had a red supergiant (RSG) phase prior to becoming LBVs.

Generally, LBVs have been considered to be stars in transition to the Wolf-Rayet stage (e.g., Maeder 1983; Humphreys \& Davidson 1994; Langer et al. 1994). Recent 
observational and theoretical work suggests that some LBVs can be the immediate progenitors of supernovae (SNe). Some corecollapse SNe may have been boosted to very high luminosity when the ejecta shock circumstellar matter expelled during a previous LBV outburst (Gal-Yam et al. 2007; Gal-Yam \& Leonard 2009; Smith et al. 2007, 2008; see Quataert \& Shiode 2012, for an alternative explanation). Groh et al. (2013) showed using stellar evolutionary models that single rotating stars with initial mass in the range of 20-25 $M_{\odot}$ have spectra similar to LBVs before exploding as SNe. This result sets the theoretical ground for low-luminosity LBVs to be the endpoints of stellar evolution. Smith (2007) discussed the possibility that the progenitor of SN 1987A, the blue supergiant Sk -69 202, was in an LBV phase when exploding. However, Sk -69 202 had a normal B supergiant spectrum prior to exploding and also had a lower luminosity, i.e., $\log \left(L / L_{\odot}\right) \sim 5.01$ (Barkat \& Wheeler 1989), than any other confirmed $\mathrm{LBV}^{1}$.

Most of the fundamental questions about the physical cause of the LBV instability are still unsolved. Outbursts with visual magnitude variations of 1-2 mag and constant bolometric luminosity are commonly referred to as classical LBV outbursts. During giant eruptions, such as PCygni in the 17th century (e.g., de Groot 1988; Lamers \& de Groot 1992) and $\eta$ Car in the 1840s (e.g., Davidson \& Humphreys 1997, 2012), the visual magnitude increases by more than 2 mag and the bolometric luminosity likely increases. The most promising explanations for the LBV instability mechanism involve radiation pressure instabilities, but also turbulent pressure instabilities, vibrations and dynamical instabilities, and binarity cannot be ruled out (Humphreys \& Davidson 1994, and references therein). The impact of binarity has especially received much attention lately (e.g., Justham et al. 2014; Smith \& Tombleson 2015; Boffin et al. 2016). There is also a debate on whether the observed radius change of the photosphere is a pulsation or outer envelope inflation driven by the Fe opacity bump (Gräfener et al. 2012; Sanyal et al. 2015).

The luminous blue variable R71 (=HD 269006) in the Large Magellanic Cloud (LMC) is presently undergoing an S Doradus outburst that started in 2005 (Gamen et al. 2009). In 2012, the star had reached unprecedented visual brightness, accompanied by remarkable variations in its optical spectrum (Gamen et al. 2012; Mehner et al. 2013). The R71 outburst is well sampled with multiwavelength spectroscopic and photometric observations and is excellent for a case study toward a better understanding of the LBV phenomenon. The star had several LBV outbursts in the last century. Between 1900 and 1950 the star experienced two contiguous outbursts (Grindlay et al. 2012; Walborn et al. 2017). Another outburst was observed between 1970-1977 (Thackeray 1974; Wolf 1975; van Genderen 1979; Wolf et al. 1981; van Genderen 1982). Currently, R71 reached $V$ magnitudes of more than one magnitude brighter than during these previous outbursts and the event was thus termed "supermaximum" by Walborn et al. (2017) ${ }^{2}$. Because of the scarce observational coverage of LBV outbursts and their potential

\footnotetext{
1 Alternative progenitor hypotheses for SN 1987A include a merger model (Podsiadlowski 1992) or an interacting winds model (Chita et al. 2008).

2 Walborn et al. (2017) mentioned an observational gap from the early 1990s to 2000. The CASPEC spectra cover this period well (see Sect. 2.1). These spectra can be matched with the same CMFGEN model as the 1984 and 2002 spectra. The star is thus at its minimum phase during this period, albeit at the brighter states of apparent microvariations.
}

importance in massive star evolution this large-amplitude event calls for special attention.

The initial years of R71's current outburst were reviewed in Mehner et al. (2013). The visual light curve of R71 reached its maximum in 2012 with $m_{\mathrm{V}, 2012}=8.7 \mathrm{mag}$, which is a brightening of about 2 mag compared to its quiescent state (Fig. 1). With small variations, the star has maintained this brightness since and shows no symptoms of an imminent end of the outburst. Its spectrum resembles a late-F supergiant with an unusually low apparent temperature for an LBV outburst of only $T_{\mathrm{eff}, 2012} \sim 6650 \mathrm{~K}$. The rise in the visual magnitude of $\mathrm{R} 71$ and the low effective temperature are unprecedented for this star.

In this second paper we cover aspects of R71's current outburst not discussed in the first paper, such as spectral and photometric oscillations and the evolution of its ultraviolet to mid-infrared spectral energy distribution (SED). We also settle the debate on its luminosity (Lennon et al. 1993; Wolf et al. 1981), which has an impact on its evolutionary path (Humphreys \& Davidson 1994). Classical LBVs have $M_{\mathrm{bol}}<$ -9.6 mag and have very likely not been RSGs. Less luminous LBVs have $M_{\text {bol }}=-8$ mag to -9 mag, lower temperatures, smaller amplitudes of their outbursts, and lower mass-loss rates. There is no strong evidence of a separation between less luminous and classical LBVs, but see Smith et al. (2004) for a discussion of a potential deficiency of LBVs in the luminosity range between them.

In Sect. 2 we describe the observations and details of the CMFGEN radiative transfer modeling and the search for periodicities in the light curve. In Sect. 3 we present the stellar parameters of R71 during quiescence, its SED, and the oscillations observed in both the light curve and the spectrum during the current outburst. In Sect. 4 we discuss its evolutionary state and the oscillatory variations. In Sect. 5 we summarize our conclusions.

\section{Observations and data analysis}

\subsection{Spectroscopic data}

In 2012 we started a spectroscopic monitoring campaign of the S Doradus outburst of R71 with X-shooter at the Very Large Telescope (VLT). X-shooter is a medium-resolution echelle spectrograph that simultaneously observes the wavelength region from 3000-24 $800 \AA$ with three arms (Vernet et al. 2011). Spectra are obtained with the narrowest available slits of $00^{\prime \prime 5}$ in the UVB arm, 0 ".4 in the VIS arm, and 0'!4 in the NIR arm yielding spectral resolving powers of $R \sim 9000-17000$. In addition, spectra with $5^{\prime \prime}$ slits in all three arms provide us with the means to achieve relative flux calibration, absolute flux calibrations to $10 \%$ accuracy, and to investigate the SED. A journal of the observations is presented in Table B.1.

Table B.1 also lists additional archival spectroscopic observations of R71 during the years 1984-2015. We obtained VLT Ultraviolet and Visual Echelle Spectrograph (UVES; Dekker et al. 2000) observations in 2002 during the quiescent state of R71 to investigate microvariations over two and a half months. The spectra cover the wavelength region from 3280-6690 $\AA$ with spectral resolving power of $R \sim 40000$. We obtained MPG ESO 2.2 m Fiber-fed Extended Range Optical Spectrograph (FEROS; Kaufer et al. 1999) observations in 2005-2015 throughout the current outburst starting at its onset. The FEROS is fed by two fibers with a $2^{\prime \prime}$ aperture for simultaneous spectra of the target and sky. The wavelength range is 3500-9200 $\AA$ and the spectral resolving power is $R \sim 48000$. Each spectroscopic data set was reduced with the corresponding 


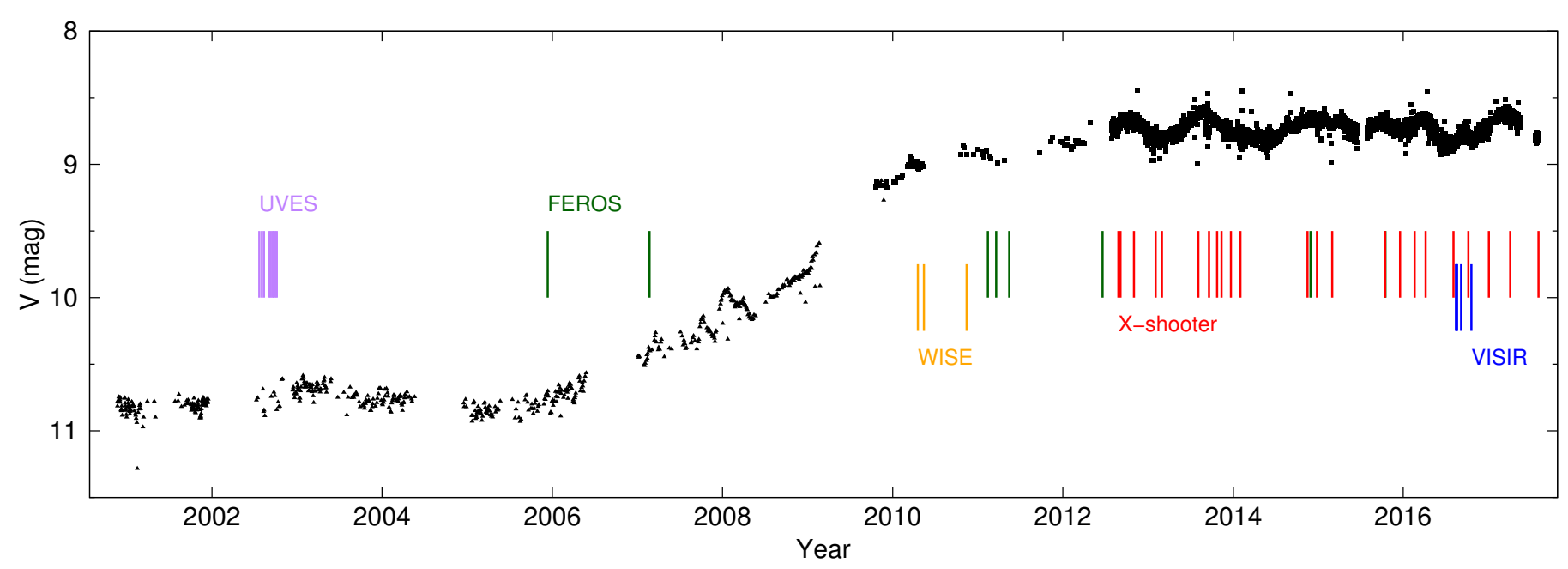

Fig. 1. Visual light curve of R71 using data from ASAS (triangles; Pojmanski 1997) and AAVSO (squares; www .aavso.org). Epochs at which spectroscopic observations with UVES, FEROS, and X-shooter are available are indicated. The epochs of WISE and VISIR mid-infrared imaging observations are also indicated. An oscillation in the light curve is clearly apparent since 2010. Variations with a shorter timescale can be seen in 2007 and 2008 during the onset of the outburst.

ESO pipeline (X-shooter pipeline version 2.8.4, UVES pipeline version 5.7.0, and FEROS pipeline version 1.0.0). The spectra were corrected for the instrumental response and atmospheric extinction but not for interstellar extinction and telluric lines. La Silla 3.6 m Cassegrain ESO Echelle Spectrograph (CASPEC; Le Luyer et al. 1979) data from 1984-1997 cover the wavelength region from $3740-5445 \AA$ with spectral resolving power of $R \sim 20000$. One La Silla 3.6 m ESO Multi-Mode Instrument (EMMI; Dekker et al. 1986) spectrum from 2000 covers the wavelength region from $6240-6870 \AA$ with spectral resolving power of $R \sim 20000$. We used International Ultraviolet Explorer (IUE; Macchetto 1976) spectra around $2800 \AA$ obtained during minimum phase in 1981, 1985, and 1986 to confirm the terminal wind velocity of R71. Enhanced data products of the Spitzer InfraRed Spectrograph (IRS; Houck et al. 2004) observations between March 2004 and March 2005 were obtained from the NASA/IPAC Infrared Science Archive. The mean full width at half maximum of the extraction profile ranges between $3^{\prime \prime}$ and $8^{\prime \prime}$.

In Mehner et al. (2013), we used the [Fe II] emission lines in the 2002 UVES spectra to determine the systemic velocity of $\mathrm{R} 71$ to $v_{\text {sys }}=192 \pm 3 \mathrm{~km} \mathrm{~s}^{-1}$. All radial velocities stated in this paper are relative to the systemic velocity.

\subsection{Photometric data}

The available photometry during the current outburst of R71 is exceptional. No other LBV outburst has been recorded with such a high temporal cadence and duration. Figure 1 shows the visual light curve of R71 since 2000 indicating the epochs of spectroscopic observations and recent mid-infrared imaging observations. The $V$-band photometry was retrieved from the All Sky Automated Survey (ASAS; Pojmanski 1997) and from the American Association of Variable Star Observers (AAVSO ${ }^{3}$ ).

The $V$-band photometry from AAVSO has been acquired since July 2012 with a frequency of 1-2 days at the Remote Observatory Atacama Desert (ROAD; Hambsch 2012) with an Orion Optics, UK Optimized Dall Kirkham 406/6.8 telescope and a FLI 16803 CCD camera and Astrodon Photometric $V$ and

\footnotetext{
3 wWw . aavso.org
}

$I_{\mathrm{C}}$ filters. Twilight sky-flat images were used for flat-field corrections. The reduction was performed with the MAXIM DL program $^{4}$ and magnitudes were determined with the LesvePhotometry program ${ }^{5}$. The magnitude error is about $0.02 \mathrm{mag}$.

Figure 2 shows the SED of R71 from the near-ultraviolet to the infrared. Photometry during the quiescent phase of the star was retrieved from many catalogs using VIZIER (Ochsenbein et al. 2000). In the mid-infrared, we complemented this data set by observations during the current outburst obtained with the upgraded VLT spectrometer and imager for the mid-infrared (VISIR; Lagage et al. 2004; Käufl et al. 2015; Kerber et al. 2016) and with the Wide-field Infrared Survey Explorer (WISE). The WISE observations of R71 occurred between April and November 2010 and the magnitudes were retrieved from the AllWISE Data Release (Cutri et al. 2013).

R71 was observed with VISIR in standard imaging mode with perpendicular nodding several times between 14 August and 19 October 2016. Three epochs in the PAH2_2 $(11.9 \mu \mathrm{m})$ filter were obtained on 14 August 2016 with 30 min on source exposure time, on 18 August 2016 with 53 min, and on 5 September 2016 with $21 \mathrm{~min}$. Another epoch in the Q1 filter $(17.7 \mu \mathrm{m})$ on August 172016 yields $10 \mathrm{~min}$ on source exposure. Finally, on October 10 2016, the star was observed in the filters: M-BAND $(4.8 \mu \mathrm{m} ; 20 \mathrm{~min})$, PAH1 $(8.6 \mu \mathrm{m} ; 5 \mathrm{~min})$, SIV $(10.5 \mu \mathrm{m} ; 2 \mathrm{~min})$, SIV_2 $(10.8 \mu \mathrm{m} ; 2 \mathrm{~min})$, PAH2 $(11.3 \mu \mathrm{m} ; 2 \mathrm{~min})$, NEII $(12.8 \mu \mathrm{m}$; $2 \mathrm{~min})$, NEII_1 (12.3 $\mu \mathrm{m} ; 2 \mathrm{~min})$, and Q2 (18.7 $\mu \mathrm{m} ; 2 \mathrm{~min})$. All observations were carried out at an airmass of 1.5 or higher, which negatively affected the image quality. For flux calibration, the science observations were either preceded or followed by a mid-infrared standard star (Cohen et al. 1999), which is the main source of uncertainty in the resulting photometry. The data reduction was performed with a custom made PYTHON pipeline and flux measurements were obtained using the custom developed IDL software package MIRPHOT (Asmus et al. 2014). The photometry was determined using both classical aperture photometry with an aperture of $1^{\prime \prime}$ and Gaussian fitting to the point source. The difference between these two methods gives an estimate of the measurement uncertainty. The systematic flux calibration uncertainty is about $10 \%$ if only one flux standard was

\footnotetext{
4 http://www. cyanogen . com

5 http://WwW. dppobservatory.net
} 


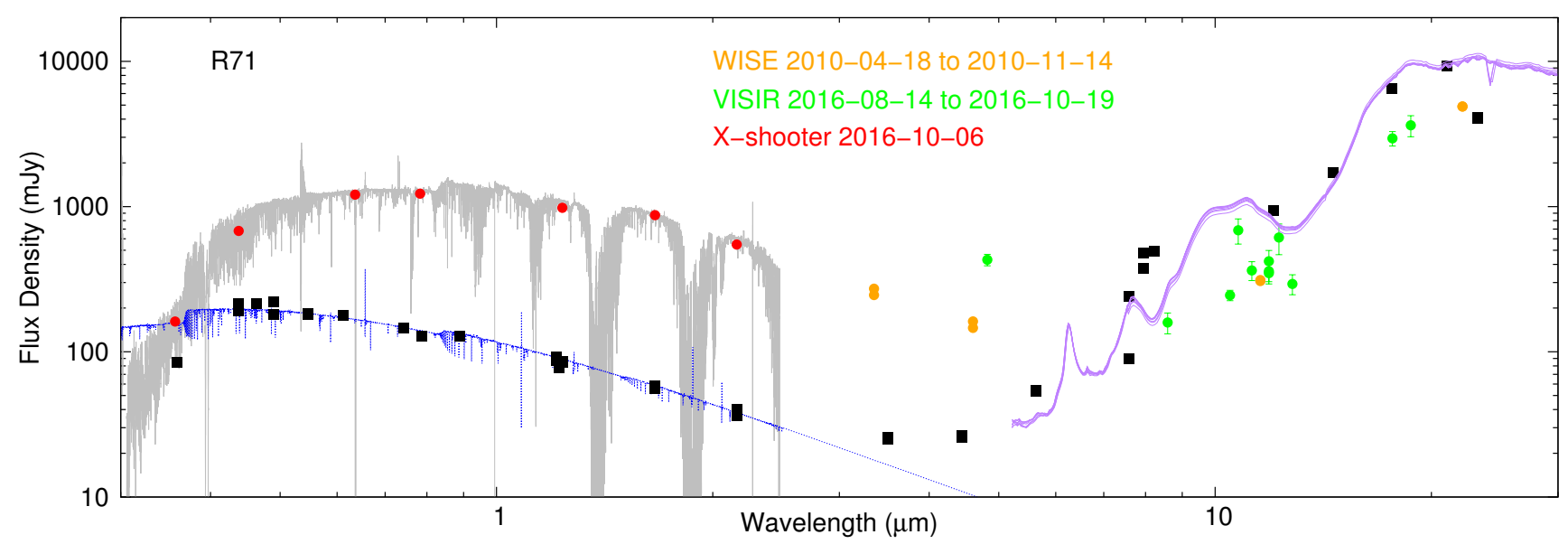

Fig. 2. Near-ultraviolet to mid-infrared SED of R71. Literature values of the quiescent state of R71 are retrieved from VIZIER (black squares). The best-fit, reddened CMFGEN model for the quiescent state of R71 is shown (blue curve), as well as the October $2016 \mathrm{X}$-shooter spectrum during outburst (gray curve) and the synthetic $U$ - to $K$-band photometry (red circles). The 2016 VISIR $M$ - to $Q 2$-band photometry (green circles) and 2010 WISE photometry (orange circles) complement the SED during the current LBV outburst of R71 to the mid-infrared. Comparison to Spitzer/IRS spectra in 2004-2005 suggests that the dust in the outermost regions has cooled down in the last decade (purple curve).

Table 1. Mid-infrared flux densities of R71 obtained from VISIR observations between 14 August and 19 October 2016.

\begin{tabular}{cccc}
\hline \hline Filter & $\begin{array}{c}\text { Wavelength } \\
(\mu \mathrm{m})\end{array}$ & $\begin{array}{c}\text { Flux density } \\
(\mathrm{mJy})\end{array}$ & $\begin{array}{c}\text { Error flux density } \\
(\mathrm{mJy})\end{array}$ \\
\hline M-BAND $^{a}$ & 4.82 & 430 & 39 \\
PAH1 & 8.59 & 159 & 26 \\
SIV $^{b}$ & 10.49 & 245 & 20 \\
SIV_2 & 10.77 & 687 & 135 \\
PAH2 & 11.25 & 363 & 54 \\
PAH2_2 & 11.88 & 375 & 32 \\
NeII_1 & 12.27 & 612 & 146 \\
NeII $^{c}$ & 12.81 & 293 & 46 \\
Q1 & 17.65 & 2948 & 338 \\
Q2 & 18.72 & 3625 & 600 \\
\hline
\end{tabular}

Notes. ${ }^{(a)}$ The individual measurements and conversion factor are all consistent with each other; we exclude a calibration problem. ${ }^{(b)}$ The source detection is very faint; only aperture photometry can be performed. ${ }^{(c)}$ The source detection is very faint; the flux is uncertain.

obtained. The resulting mid-infrared spectral flux densities from the 2016 VISIR images of R71 are listed in Table 1.

\subsection{Radio data}

R71 was observed at radio wavelengths with the Australia Telescope Compact Array at two different epochs in 2014 and 2015; see Table 2. The observations were performed with the array in the most extended configuration $(6 \mathrm{~km})$ and the Compact Array Broadband Backend (CABB) " $4 \mathrm{~cm}$ " receiver. The receiver bandwidth was split in two $2 \mathrm{GHz}$ sub-bands centered at $5.5 \mathrm{GHz}$ and $9 \mathrm{GHz}$ with $2048 \times 1 \mathrm{MHz}$ channels in each subband. R71 was observed with 15 min scans on target, alternating with $2.5 \mathrm{~min}$ on the phase calibrator ICRF J052930.0-724528. The scans were distributed over 12 values of hour angle to achieve good $u v$ coverage. The total integration times are listed in Table 2. For bandpass and flux calibrations, observations of ICRF J193925.0-634245 were performed on each date.
The data were reduced separately for each central frequency (5.5 and $9 \mathrm{GHz}$ ) with the MIRIAD package (Sault et al. 1995). The data reduction process consisted in flagging bad data and calculating bandpass corrections and complex gain solutions. The calibrated visibilities were imported in the Common Astronomy Software Applications (CASA) package v5.0.0 (McMullin et al. 2007) and imaged with the task tclean. A natural weighting scheme for the visibilities was adopted to preserve the sensitivity. For the deconvolution of the dirty image, the algorithm of Högbom (1974) was used. Table 2 summarizes the properties of the cleaned radio maps. The rms noise in the residual maps is less than a factor of two larger than the theoretical noise, meaning that the sensitivity is mostly limited by statistical noise. R71 is not detected. Upper limits on the flux density at the radio frequencies are provided in the last column of Table 2.

\subsection{CMFGEN radiative transfer modeling}

The stellar winds of LBVs can be so dense that many emission lines form in the wind and veil the underlying photospheric spectrum. The radiation emitted by the stellar surface interacts with the optically thick, dense wind, making the analysis of the emerging stellar spectrum challenging. In addition to temperature, luminosity, and effective gravity, other quantities such as mass-loss rate, microturbulence, and wind acceleration law affect the morphology of the spectrum. Complex radiative transfer models, which include the necessary physics to study the radiation transport across the atmosphere and wind, are needed to obtain realistic parameters.

We analyzed pre-outburst spectra of R71 with atmosphere models computed with the radiative transfer code CMFGEN (version 5 May 17, Hillier \& Miller 1998). The CMFGEN code comprises the state of the art in non-local thermodynamic equilibrium (non-LTE) radiative transfer and has been successfully applied to several LBVs (e.g., Hillier et al. 2001; Groh et al. 2006, 2009, 2011; Clark et al. 2009, 2012; Georgiev et al. 2011). In CMFGEN, each model is defined by the effective temperature, luminosity, mass-loss rate, wind volume filling factor, wind terminal velocity, effective gravity (or stellar mass), and abundances. For a review on the essential properties of the code and 
Table 2. Summary of ATCA radio observations and radio map properties.

\begin{tabular}{|c|c|c|c|c|c|c|c|}
\hline Date & $\begin{array}{l}\text { Frequency } \\
(\mathrm{GHz})\end{array}$ & $\begin{array}{l}\text { Integration time } \\
(\min )\end{array}$ & $\begin{array}{l}\text { Synthesized beam } \\
\text { HPBW (arcsec) }\end{array}$ & $\begin{array}{l}\text { Position angle } \\
\text { (deg) }\end{array}$ & $\begin{array}{l}\text { Largest angular scale } \\
\text { (arcsec) }\end{array}$ & $\begin{array}{l}\text { RMS noise } \\
\left(\mu \mathrm{Jy}_{\text {beam }}^{-1}\right)\end{array}$ & $\begin{array}{l}F_{v}{ }^{a} \\
(\mu \mathrm{Jy})\end{array}$ \\
\hline 2014 Feb. $14-16$ & 5.5 & 135 & $2.33 \times 1.76$ & -12.2 & 100 & 13 & $<39$ \\
\hline 2014 Feb. $14-16$ & 9.0 & 135 & $1.35 \times 1.17$ & -18.5 & 50 & 26 & $<78$ \\
\hline 2015 Mar. 2-4 & 5.5 & 180 & $2.05 \times 1.79$ & -3.6 & 50 & 8 & $<24$ \\
\hline 2015 Mar. 2-4 & 9.0 & 180 & $1.49 \times 1.32$ & -12.8 & 30 & 9 & $<27$ \\
\hline
\end{tabular}

Notes. Project ID: C1973. ${ }^{(a)}$ R71 is not detected. Upper limits on the flux density at the radio frequencies.

the basics of spectroscopic analysis of massive stars using photospheric and wind diagnostics, see, for example, Groh (2011) and Martins (2011). The following diagnostics were used to find the best-fitting stellar model for R71:

- Effective temperature. The effective temperature is obtained using the ionization balance of different ionization stages of the same chemical species. The best line diagnostic for R71 and our spectral coverage is the Si ionization balance using the lines Si II $\lambda 6347$; Si III $\lambda \lambda 4553,4568,4575$; and Si IV $\lambda \lambda 4089,4116$. We also use the Mg II $\lambda \lambda 4481,6343$ lines. For both elements, we assume LMC abundance. LMC abundances are scaled to the solar abundances from Asplund et al. (2009), assuming $Z_{\odot}=0.014$ and $Z_{\mathrm{LMC}}=0.006$ (Yusof et al. 2013).

- Luminosity and extinction. The bolometric luminosity and extinction are obtained self-consistently by fitting CMFGEN model atmosphere spectra to the observed optical to near-infrared SED during the quiescence phase of the star. The distance to the LMC is well-determined and the errors associated with this method depend largely on the quality of the photometry. We adopted a distance to the LMC of $49.97 \pm 0.19$ (statistical) \pm 1.11 (systematic) $\mathrm{kpc}$ (Pietrzyński et al. 2013). We iteratively matched the colors and fluxes of the $J H K$ photometry to those synthesized from reddened CMFGEN models. Because the extinction in the near-infrared is low, we avoid large uncertainties in the luminosity, which may arise from an uncertain ratio of extinction to reddening $R_{V}$. To uncover a potential unusual extinction curve toward $\mathrm{R} 71$, we also determine the ratio of extinction to reddening (Sect. 3.2).

- Gravity and mass. The wings of the hydrogen Balmer and Paschen lines are the main diagnostics for the gravity, $\log g$.

- Mass-loss rate and clumping. Wind clumping has a significant impact on obtaining the mass-loss rates of massive stars (Hillier 1991; Fullerton et al. 2006; Puls et al. 2008; Sundqvist \& Owocki 2013; Sundqvist et al. 2014). The CMFGEN code allows for the presence of clumping using a volume-filling approach, assuming that material is unclumped close to the star and reaches maximum clumpiness at large distances. In principle, the mass-loss rate and clumping could be constrained from the emission strengths and electron scattering wings of recombination lines such as $\mathrm{H} \alpha$ and higher Balmer lines. However, we lack precise response curves for the pre-outburst spectroscopic data and thus cannot constrain the clumping. We therefore use a volumefilling factor of $f=1(\bar{\rho}=f \rho$, where $\bar{\rho}$ is the homogeneous unclumped wind density). The mass-loss rate scales with $\dot{M}=$ const. $\sqrt{f}$. Accordingly, the derived mass-loss rates are upper limits.

- Surface abundance. It is essential to determine the abundance of $\mathrm{He}$ and $\mathrm{CNO}$ elements to constrain the history and evolutionary state of R71. LBVs show usually an overabundance of $\mathrm{He}$ and $\mathrm{N}$, and a depletion of $\mathrm{H}, \mathrm{C}$, and $\mathrm{O}$. The He abundance is determined from the non-diffuse He I lines at $\lambda \lambda 3965,4121$, and $4713 \AA$, but also taking into account other helium lines. $\mathrm{C}$ and $\mathrm{N}$ abundances are derived using the numerous $\mathrm{C}$ and $\mathrm{N}$ lines present in the optical range; these include N II $\lambda \lambda 3995,4601-4643,5667-$ 5711,6482; N III $\lambda \lambda 4196,4216,4511,4515,4523,4602$, 4907; C II $\lambda \lambda 4267,6578,6583$; and C III $\lambda \lambda 4070,4153-56-$ $63,4326,5305,6205$. The $\mathrm{O}$ abundance is estimated using O I $\lambda \lambda 7772-7775$. We only determine the abundances of $\mathrm{H}, \mathrm{He}$, and CNO. For all other elements we assume LMC abundances. As above for the effective temperature, solar abundances are from Asplund et al. (2009) and the LMC abundances are scaled to the solar abundances, assuming $Z_{\odot}=0.014$ and $Z_{\mathrm{LMC}}=0.006$.

- Microturbulence. Line broadening reflects the effects of both rotation and turbulence. Microturbulence, $v_{\text {turb }}$, can have a considerable effect on the abundances. We constrain $v_{\text {turb }}$ using the Si III lines at $\lambda 4560 \AA$. A large effect can be observed for the He I $\lambda \lambda 6678,7065$ lines, where, with lower microturbulence, the red line wings move blueward.

- Wind terminal velocity. We determine a lower limit to the terminal velocity, $v_{\infty}$, in optical spectra using the velocity of the maximum absorption of hydrogen and helium lines. We validated this estimation using the metallic resonance line Mg II 2803 in IUE spectra obtained in 1981, 1985, and 1986 during quiescent state.

\subsection{Search for periodicities in the light curve}

The AAVSO $V$-band light curve during the outburst of R71 (since 2012) was analyzed to search for periodicities using the Lomb-Scargle technique (Lomb 1976; Scargle 1982). The Lomb-Scargle periodogram is a commonly used statistical tool designed to detect periodic signals in unevenly spaced observations. A heuristic was used to determine the frequency range and grid spacing, resulting in a period range of $\sim 0.2-20000 \mathrm{~d}$ and grid spacing of $0.00010893 \mathrm{~d}^{-1}$. The analysis was performed using the astropy.stats.LombScargle package.

We generated 1000 simulated light curves via a bootstrap technique. Bootstrapping involves sampling with replacement from the original data set. Magnitude-time data pairs are selected at random from the original light curve with multiple selections possible. Multiple selections of the same pairs are then discarded, leaving us with light curves made up of $63 \%$ of the original data. Lomb-Scargle analysis was performed on each of these light curves in the same manner as on the original light curve.

\section{Results}

\subsection{Current $S$ Doradus outburst of $R 71$}

We analyzed changes in the near-ultraviolet to mid-infrared brightness of R71 during its current S Doradus outburst. We 
also compared several spectral features between 1984 and 2017. The spectra until 2005 cover the quiescence phase of R71; see Fig. 1. Small variations in the line profiles occur during quiescence phase due to microvariations, which are also observed in the photometry, but the spectra closely resemble each other. At the time of the 2005 FEROS spectrum, the current outburst commenced but without major changes in the spectrum yet. The 2007 FEROS spectrum is a snapshot of the transitional phase between the quiescent and eruptive state. The FEROS spectra since 2011 and the X-shooter spectra since 2012 show R71 in outburst, when the visual magnitude had reached its maximum visual brightness.

\subsubsection{Spectral energy distribution}

Figure 2 compares the pre-outburst near-ultraviolet to midinfrared SED of R71 with its SED during the current S Doradus outburst. During the outburst, the spectral energy density of R71 increases dramatically from about $3500 \AA$ to $6-7 \mu \mathrm{m}$. Blueward of $3500 \AA$, the star becomes fainter. This is the usual behavior seen in S Doradus outbursts, i.e., the flux at ultraviolet wavelengths is reprocessed to longer wavelengths as the effective temperature of the photosphere decreases. The bolometric luminosity likely remains constant.

Redward of about $7 \mu \mathrm{m}$, where the mid-infrared excess from surrounding cold dust dominates, lower flux is observed in 2010 and 2016 compared to 2004-2005. This suggests cooling of the dust in the outermost regions. The VISIR and WISE observations suggest that the emission bump around $10 \mu \mathrm{m}$ has disappeared. This indicates that grain evolution is occurring and that we are possibly seeing grain growth (e.g., Kessler-Silacci et al. 2006).

In $M$ band $(\lambda 4.82 \mu \mathrm{m})$ we find an increase of hot dust emission between the WISE observations in 2010 and the VISIR images in 2016. Since the individual measurements and conversion factor for the VISIR observations are consistent with each other, we exclude a calibration problem. The increase in $M$ band may thus be the result of newly formed dust caused by the current outburst. In this case, we expect an increase at longer wavelengths in the next years. Two of the VISIR photometric points (SIV_2, NEII_1) exhibit significantly higher fluxes than other simultaneously recorded filters at nearby wavelengths. As the same calibrator stars are used and their cross-calibration is consistent, calibration issues can be excluded. Future low-resolution VISIR spectra would resolve the issue.

With respect to the oscillatory variations discussed in Sect. 3.1.3 we find that during the brighter phases in $V$ the color $V-I$ is smaller, i.e., the star becomes bluer. Synthetic photometry of the X-shooter spectra with wide $5^{\prime \prime}$ slits confirms this for all wavelengths. The $V-I$ color varies by about \pm 0.065 mag from the average value $V-I \approx 0.558 \mathrm{mag}$. This results in temperature variations of about $500 \mathrm{~K}$ between the (local) minima and maxima (changes in spectral type between F5 and F9; Pecaut \& Mamajek 2013). The associated radius variation is less than $10 \%$.

\subsubsection{Hydrogen and helium lines}

Figure 3 shows a time series of hydrogen and helium lines from 1984 to 2017. H $\alpha$ changed from a P Cyg profile with broad emission wings to a prominent double-peaked profile by 2011. In 2007 extra emission compared to the continuum is observed indicating an increased mass-loss rate. A strong blueshifted absorption component at $v \approx-120 \mathrm{~km} \mathrm{~s}^{-1}$ is present since November 2014. One could debate if this may have been caused by a shell ejection or a time-dependent wind, perhaps triggered by the bistability mechanism (Groh \& Vink 2011). Assuming that the extra $\mathrm{H} \alpha$ emission observed in 2007 may have been the signature of a mass-ejection event, a corresponding shell of material may now be at about $250 \mathrm{au}$. The helium absorption lines disappear as the star becomes cooler. There is no evidence of He II emission during its quiescent state, which could have indicated a wind-wind interaction with a potential close companion.

\subsubsection{Oscillatory variations in the light curve and spectrum}

During the current outburst of R71, oscillatory variations are observed in its optical light curve and spectrum (Figs. 1, 4, and 6). These variations were also mentioned by Walborn et al. (2017), who determined a periodicity of $445 \pm 40 \mathrm{~d}$. The light curve during outburst shows crudely sinusoidal variations between fainter and brighter states. In Sect. 3.1.1 we already described the $V-I$ color variations during these oscillations; from the color variations we infer temperature variations of about $500 \mathrm{~K}$ and radius variation of less than 10\%. In 2007 and 2008, when the brightness of the star was increasing, the variations are observed but with shorter timescales.

Figure 5 shows the Lomb-Scargle periodogram of the $V$-band light curve of the star from 2012-2017. The periodogram reveals strong peaks at frequencies corresponding to periods of about $0.2 \mathrm{~d}, 0.5 \mathrm{~d}, 1.0 \mathrm{~d}$, and $425 \mathrm{~d}$. The three shortest periods correspond to the duration of the observation, the nightly cadence, and half of the nightly cadence. Lomb-Scargle analysis was performed on 1000 bootstrapped light curves in the same manner as on the original light curve. In all instances, a period at $425 \mathrm{~d}$ was recovered, indicating that this value is robust and significant at a $\gtrsim 3$-sigma level. While the light curve and this analysis indicate pseudo-steady oscillations, these are to some extent chaotic, which points toward a phenomenon within the extended atmosphere. A quantitative estimate of the period error is not possible and would be meaningless to some extent, as we cannot distinguish between a random error and systematic evolution of the period during the time span of the observations.

Spectroscopic variations manifest themselves in that absorption lines vary between single and double on a timescale of about $850 \mathrm{~d}$, i.e., twice the length of the photometric cycle. The uncertainty of the spectroscopic cycle length is about $50 \mathrm{~d}$, which is limited by the sparsity of spectroscopic epochs. The spectroscopic variation can best be observed in the many Fe I absorption lines (Fig. 4). The bluer of the two absorption lines is at a velocity of about $-70 \mathrm{~km} \mathrm{~s}^{-1}$. The right panel of Figs. 4 and 6 correlate the radial velocity of the blue wing of the Fe I absorption with the $V$-band magnitude of the star. Three spectroscopic cycles have been observed since 2011. The spectra in 2011 have two absorption components, which become single by June 2012. In November 2012, the second absorption component reappears and then disappears again by November 2014, etc. The cycle length is twice as long as the photometric cycle, hinting at period doubling; see Sect. 4.2.

\subsubsection{Low-excition emission nebula and dust shell}

In 2012, many low-excitation metal emission lines appeared and became stronger (Mehner et al. 2013; see also the Fe I line indicated in the middle panel of Fig. 3). Mehner et al. (2013) proposed that these lines indicate the presence of a neutral nebula 

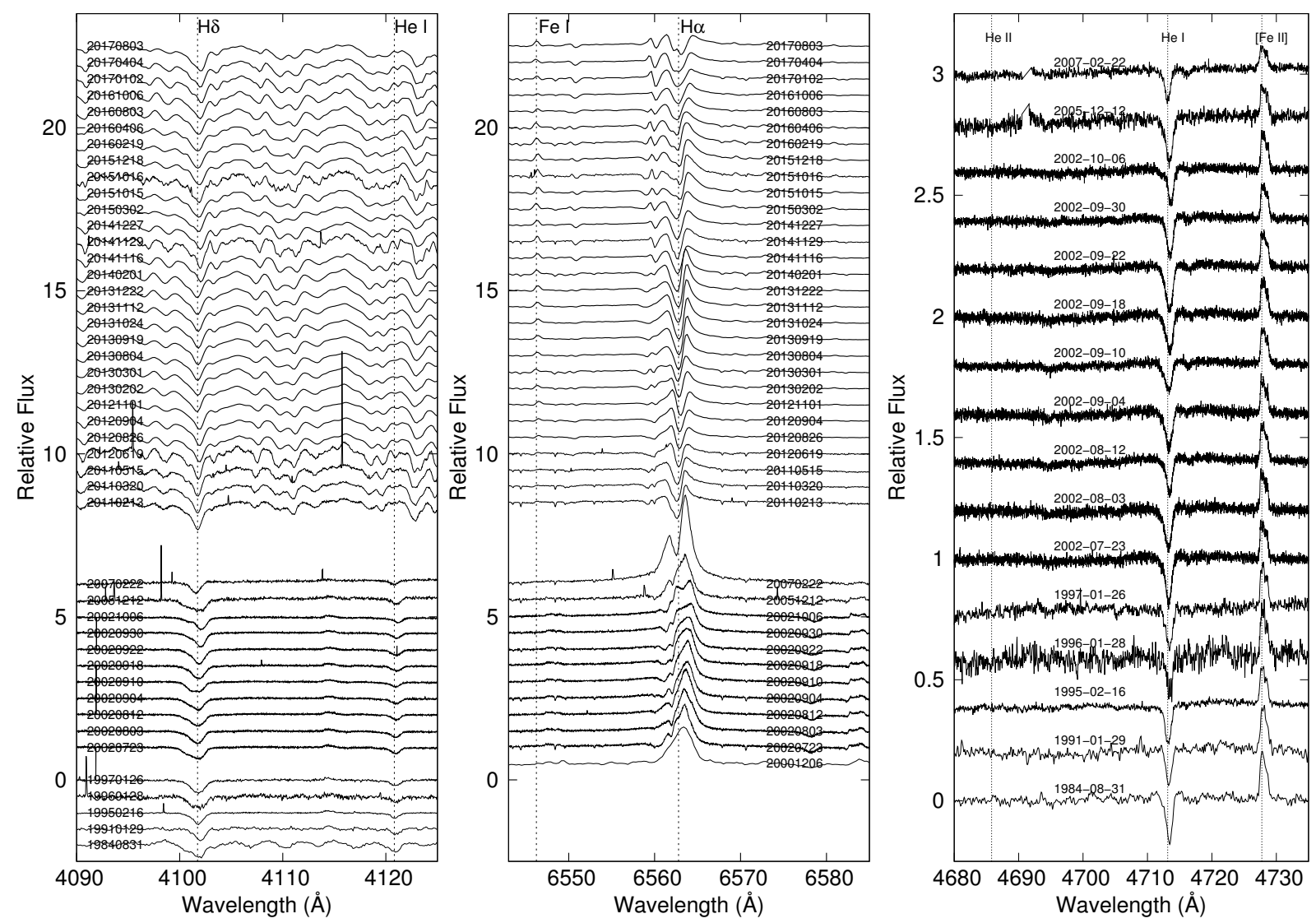

Fig. 3. Left and middle panel (common vertical timescale): $\mathrm{H} \delta$ and $\mathrm{H} \alpha$ in $\mathrm{R} 71$ from 1984 to 2017. The $\mathrm{H} \alpha$ P Cyg profile changed to an inverse double-peaked symmetric profile during the outburst. Until 2007, the star is hot and increased line emission implies an increased amount of circumstellar material. An absorption component close to system velocity dominates the profile during the outburst phase and several absorption components trace the line-of-sight structure. An example of a low-excitation emission Fe I line is shown. Right panel: He II 4686 and He I 4713 in R71 from 1984 to 2007. No He II emission is present. The continuum is normalized to unity, velocities are in the R71 rest frame.

with an expansion velocity on the order of a few $\mathrm{km} \mathrm{s}^{-1}$, likely ejected during the current outburst. However, it now seems more likely that these emission lines are due to dynamical processes in the extended envelope. This is supported by the evolution observed in metal lines such as Fe II from P Cyg profiles to inverse P Cyg profiles (Walborn et al. 2017), which has also been seen for the LBV S Doradus (Wolf \& Stahl 1990).

Before the current eruption R71 already showed nebular lines, such as [N II] (Stahl \& Wolf 1986), and strong mid-infrared radiation (Glass 1984; Wolf \& Zickgraf 1986; Voors et al. 1999), which are evidence of a dust shell, but Weis (2003) found no extended optical nebula in the HST/WFPC2 image of R71 obtained in 1998. If R71 formed an optical LBV nebula during its 1970 s outburst, it is either extremely faint or very small $\left(<0.5^{\prime \prime}\right)$. Guha Niyogi et al. (2014) extensively discussed the infrared spectral features of R71 and derived dust shell parameters, composition, and mass. They modeled the optical-toinfrared SED of R71 and derived an outer radius of the dust shell of $6 \times 10^{4}$ au (1.2" on sky). In case of a mass ejection event in 2007 , the resulting shell would be at a distance of about 250 au from the star (5 mas on sky at the distance of the LMC) and thus would only be detectable with high-angular resolution techniques such as interferometry (see Fig. 1 in Beltrán \& de Wit 2016).

The mid-infrared VISIR images suffer from bad image quality, mainly because of the high airmass, but also because of large seeing values during the observations, making the detection of a small nebula difficult. The best image quality was achieved in the PAH2_2 image on 2016-08-18 and in Q1 on 2016-08-17. In both images the source has a featureless roundish extent with a FWHM of $\sim 1^{\prime \prime}$ (in agreement with the outer dust radius of 1.2" derived by Guha Niyogi et al. 2014). Because of the uncertainty in image degradation with airmass due to a degraded chopping performance of the telescope, this value should be regarded as an upper limit to the true size of the object. No evidence of further extended emission is found in any of the images.

The IRS spectra in 2004 and 2005 (Fig. 2) show that the 8$12 \mu \mathrm{m}$ wavelength range is dominated by a strong silicate emission feature, demonstrating the presence of an optically thin or clumpy dust shell heated by the star. A weaker corresponding silicate feature is present at $18 \mu \mathrm{m}$ and polycyclic aromatic hydrocarbon (PAH) emission is visible at $6.3 \mu \mathrm{m}, 7.7 \mu \mathrm{m}$, and $11.3 \mu \mathrm{m}$. Evolution of these mid-infrared features indicates that we are witnessing grain evolution; see Sect. 3.1.1.

The LBV nebulae are often ionized and free-free emission is observed at radio wavelengths. Successful observations of extended LBV nebulae in the LMC with ATCA have been reported (Agliozzo et al. 2012). However, R71 is not detected in 20142015 ATCA radio maps. To explain this non-detection, we derive the radius $R_{\mathrm{S}}$ of the Strömgren sphere for ionized hydrogen in a nebula surrounding R71 during the quiescent phase (as the ionizing source). We adopt an ultraviolet photon flux of $\log \left(N_{\mathrm{UV}}\right) \sim 45.5$ (the value for a B3 supergiant; Panagia 1973 ) and assume a typical electron density of $100 \mathrm{~cm}^{-3}$ for an LBV nebula. This results in $R_{\mathrm{s}} \approx 0.2 \mathrm{pc}\left(0.9^{\prime \prime}\right.$ on sky). A similar HII region, in thermal equilibrium with electron temperature 

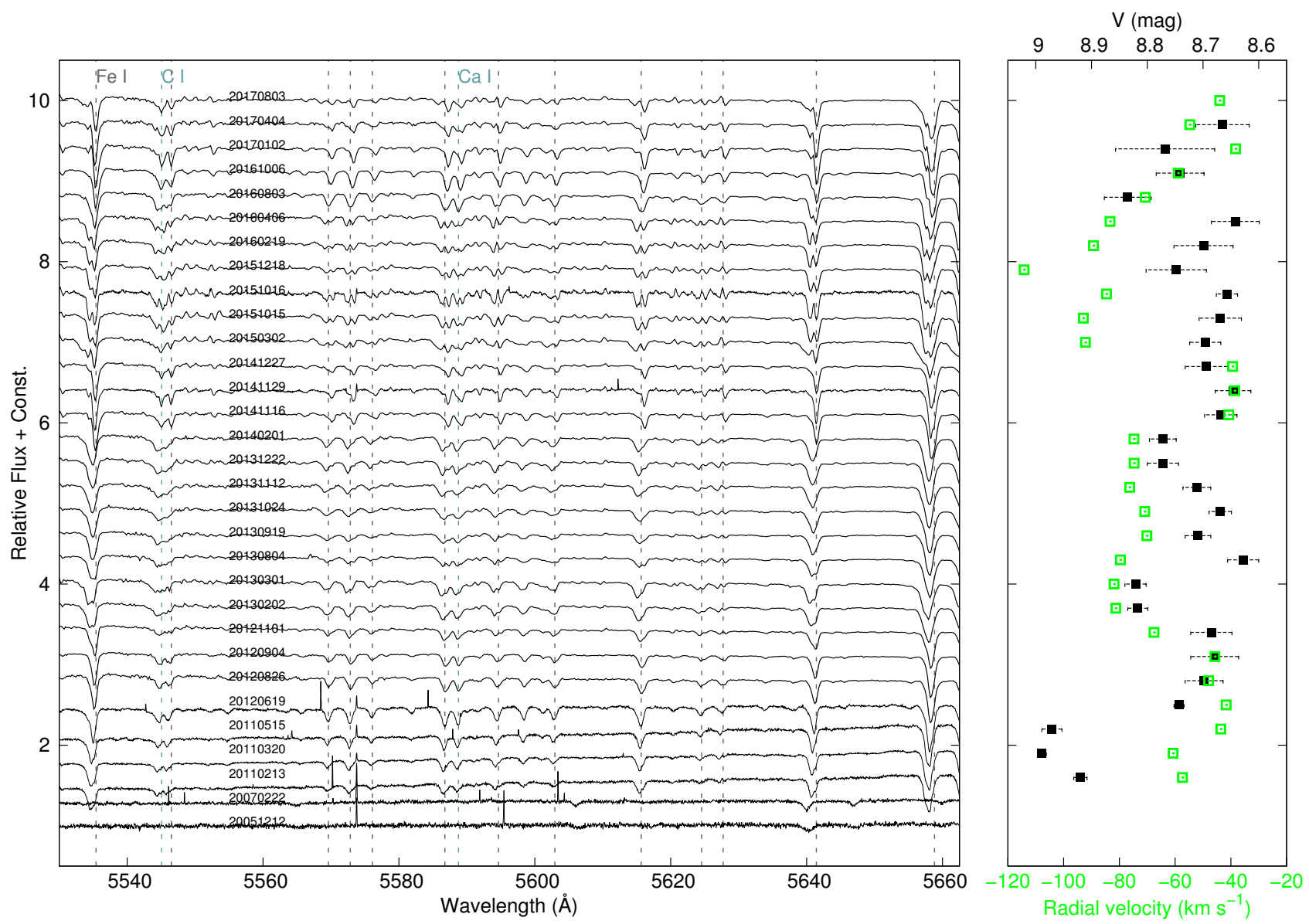

Fig. 4. Left panel: absorption lines develop a second blue component with a cycle of about $850 \mathrm{~d}$. Three cycles have been observed so far; see also Fig. 6. Gray dotted lines indicate Fe I lines. Right panel: $V$-band magnitude (black squares) at the time of the spectra to the left (average of $\pm 1 \mathrm{~d}$ around the time of the spectrum). The radial velocity of the blue wing with respect to the red wing of the line is shown (green squares); measurements were performed at the midpoint (between maximum and minimum depths of the lines).

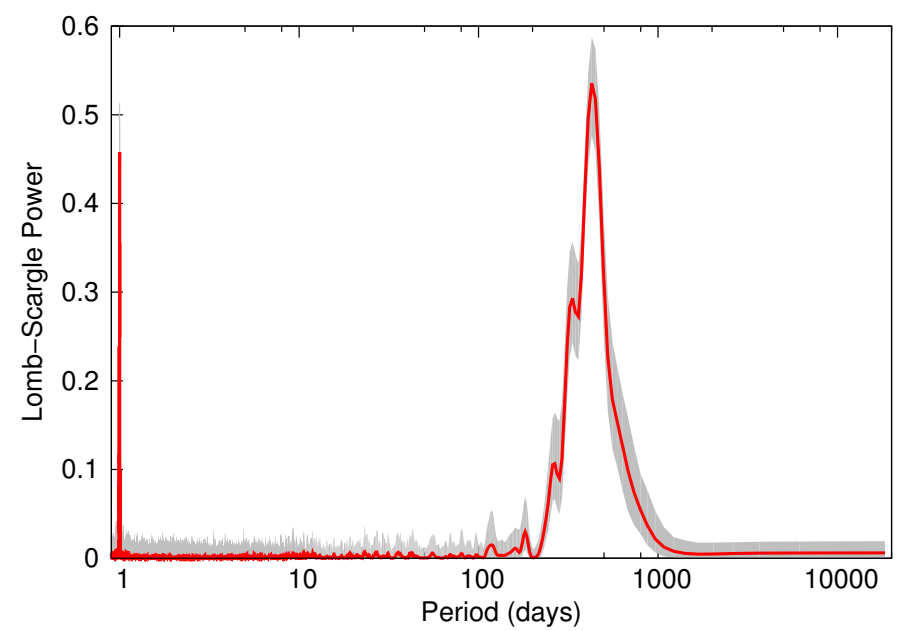

Fig. 5. Lomb-Scargle periodogram of R71's $V$-band light curve from 2012-2017. The gray shaded contours represent the range of LombScargle powers obtained with 1000 simulated light curves. A period of $425 \mathrm{~d}$ is found.

of $T_{\mathrm{e}} \approx 10^{3}-10^{4} \mathrm{~K}$, produces flux densities on the order of $1 \mathrm{mJy}$ at $9 \mathrm{GHz}$ and would be detected with ATCA. We deduce that the ionized region in the ejecta of R71 must thus be much smaller than $<0.35^{\prime \prime}$ to escape detection with the achieved sensitivities. For higher electron densities the ionized region would

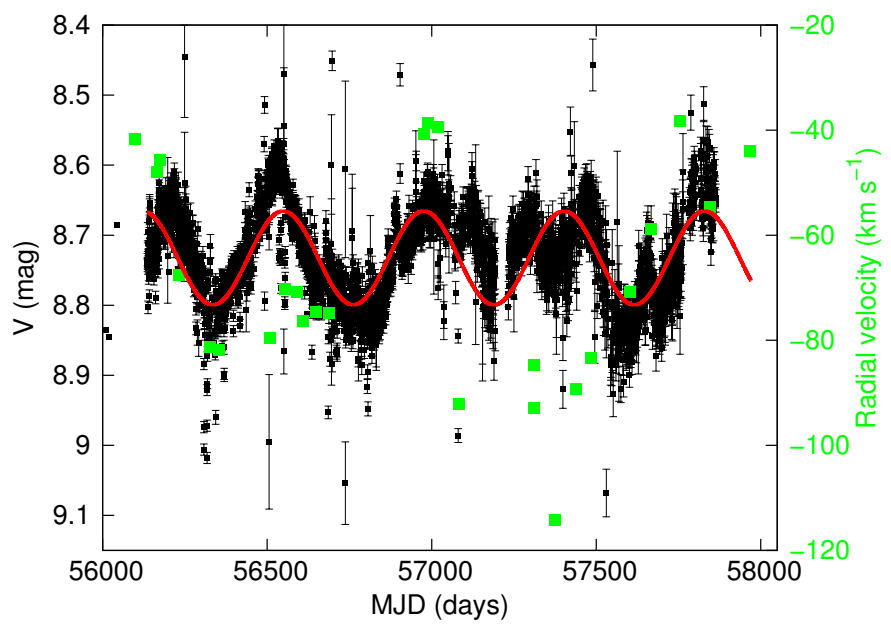

Fig. 6. R71 $V$-band magnitude (black squares) and radial velocities of the blue wing with respect to the red wing of the double line profiles (green squares) are shown. A sinusoidal curve with a period of $425 \mathrm{~d}$, corresponding to the peak in the periodogram, is superposed in red. The photometric cycle is half the length of the spectroscopic cycle with some chaotic behavior. This may hint at period doubling; see Sect. 4.2.

be even smaller, but brighter. For sufficiently high electron densities $\left(\approx 5 \times 10^{4} \mathrm{~cm}^{-3}\right)$, it is possible that hydrogen recombined since the beginning of the outburst. 


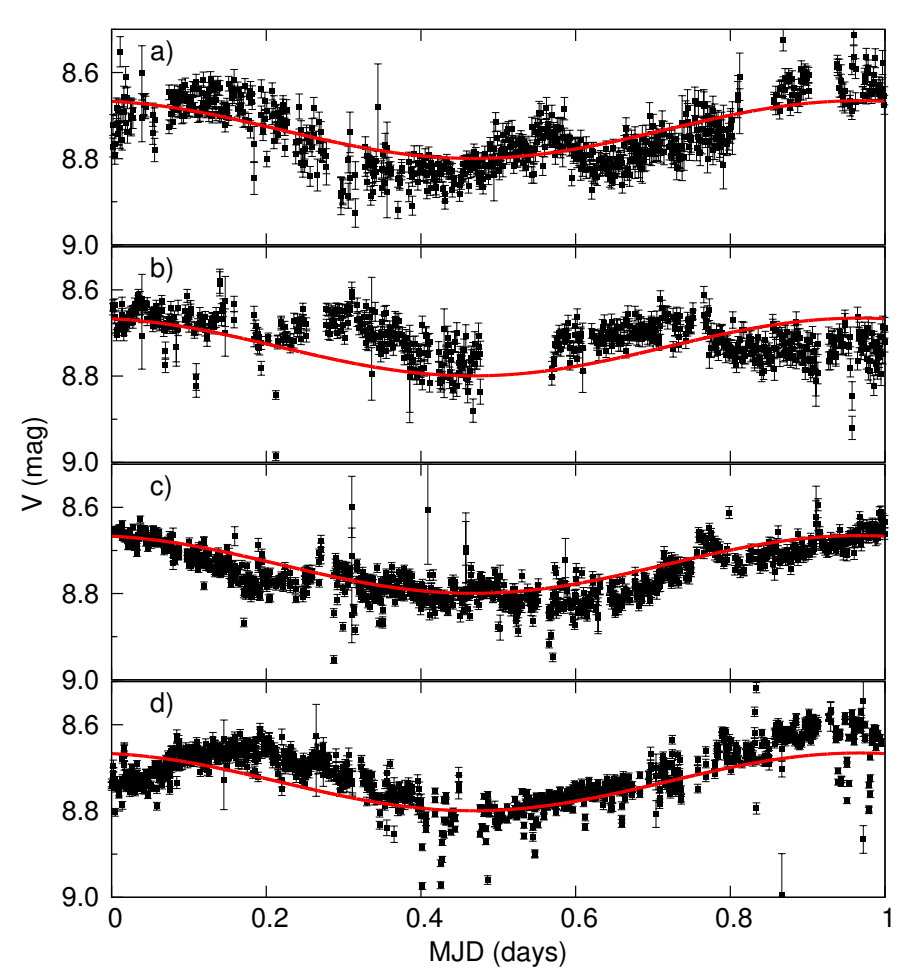

Fig. 7. R71 $V$-band magnitude (black squares) folded in phase space with a cycle of $425 \mathrm{~d}$. A sinusoidal curve with a period of $425 \mathrm{~d}$ is shown in red. Cycle a) corresponds to MJD 57417-57 844, cycle b) to MJD 56990-57417, cycle c) to MJD 56564-56990, and cycle d) to MJD $56135-56564$.

\subsection{Extinction and reddening toward $R 71$}

The extinction and reddening toward an object depend on the wavelength, the amount of dust along the line of sight, and the dust grain sizes and composition. The value of the ratio of extinction to reddening $R_{V}=A_{V} / E(B-V)$ implies the size of the dust grains. The Galactic interstellar dust in the line of sight to the LMC does not significantly affect the spectrum of R71 (Fitzpatrick 1986) and its spectral classification can thus be used to measure the amount of reddening and extinction.

We used $B$ - to $R$-band photometry from Ducati (2002), $J$ to $K$-band photometry from Cutri et al. (2003), and for the true magnitudes of the star in these filters we synthesized photometry from our best-fit unreddened CMFGEN model. Figure 8 shows the linear fit to the extinction curve resulting in $R_{V}=$ $4.1 \pm 0.5$, which is higher than the average of $R_{V}=3.4$ for the LMC (Gordon et al. 2003). However, the extinction curve suggests that there is no abnormal reddening. We lack precise simultaneous multiwavelength photometry of the quiescent state of the star to confidently determine the $R_{V}$ value and thus assume the average LMC value. However, a value of $R_{V}$ different from average may not be unexpected for a star that has undergone several S Doradus phases accompanied by high mass-loss (Lennon et al. 1993; Voors et al. 1999). Unusual grain size and dust distribution have, for example, also been observed for $\eta \mathrm{Car}$ (Mitchell \& Robinson 1986; Hillier \& Allen 1992) and AG Car (Groh et al. 2009).

The process to derive the reddening and extinction toward $\mathrm{R} 71$ is iterative, as mentioned in Sect. 2.4. Figure 9 shows that our best-fit CMFGEN model spectrum reddened by $E(B-$ $V)=0.18 \mathrm{mag}$ and $R_{V}=3.4$ according to the Fitzpatrick law (Fitzpatrick 1999) matches the observed photometry best.

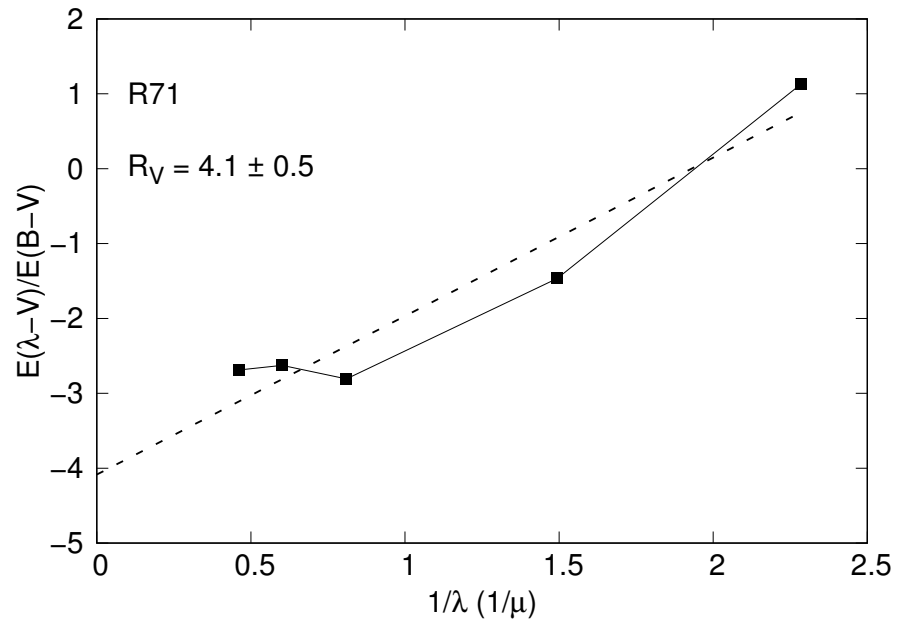

Fig. 8. Linear fit to the extinction curve of R71. $B$ - to $R$-band photometry were taken from Ducati (2002) and $J$ - to $K$-band photometry from Cutri et al. (2003). For the true magnitudes of the star in these filters, we synthesized photometry from our best-fit unreddened CMFGEN model (gray curve in Fig. 9). The resulting ratio of extinction to reddening is $R_{V}=4.1 \pm 0.5$.

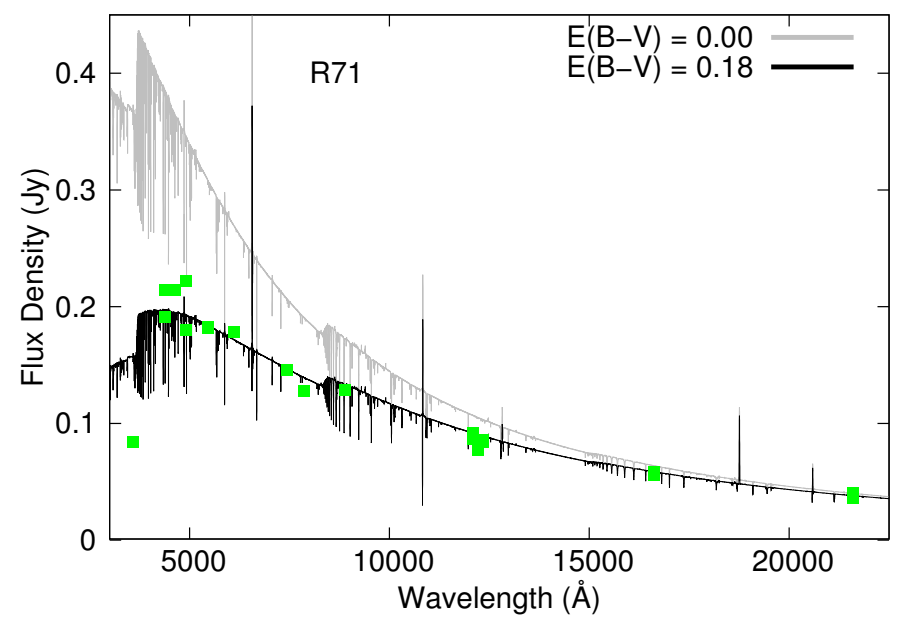

Fig. 9. Best-fit CMFGEN atmosphere model compared to the opticalto-near-infrared photometry of R71. The best match with the photometry (green squares, retrieved from VIZIER) is found for $E(B-V)=$ 0.18 mag. The three outliers at wavelengths blueward of $5000 \AA$ are ignored since these most likely correspond to photometry obtained in phases where the star is not at its quiescent state.

This results in an extinction value of $A_{V}=0.6 \pm 0.1 \mathrm{mag}$. An extinction value of $A_{V}=0.6 \mathrm{mag}$ is similar to that found by Lennon et al. (1993), who derived $A_{V}=0.63 \mathrm{mag}$, and much higher than the values found by Wolf et al. (1981) and van Genderen et al. (1988), who derived $A_{V}=0.15 \mathrm{mag}$ and $A_{V}=0.37 \mathrm{mag}$, respectively. These authors assumed much smaller reddening values and thus found lower effective temperatures and lower luminosities; this is not consistent with our CMFGEN models of the spectra during quiescence. This has implications for the luminosity of the star and thus its evolutionary past; see Sect. 4.1.

\subsection{Fundamental stellar wind parameters in quiescence state}

We used pre-outburst spectra obtained in 1984-2005 and CMFGEN atmosphere modeling to determine the classical 


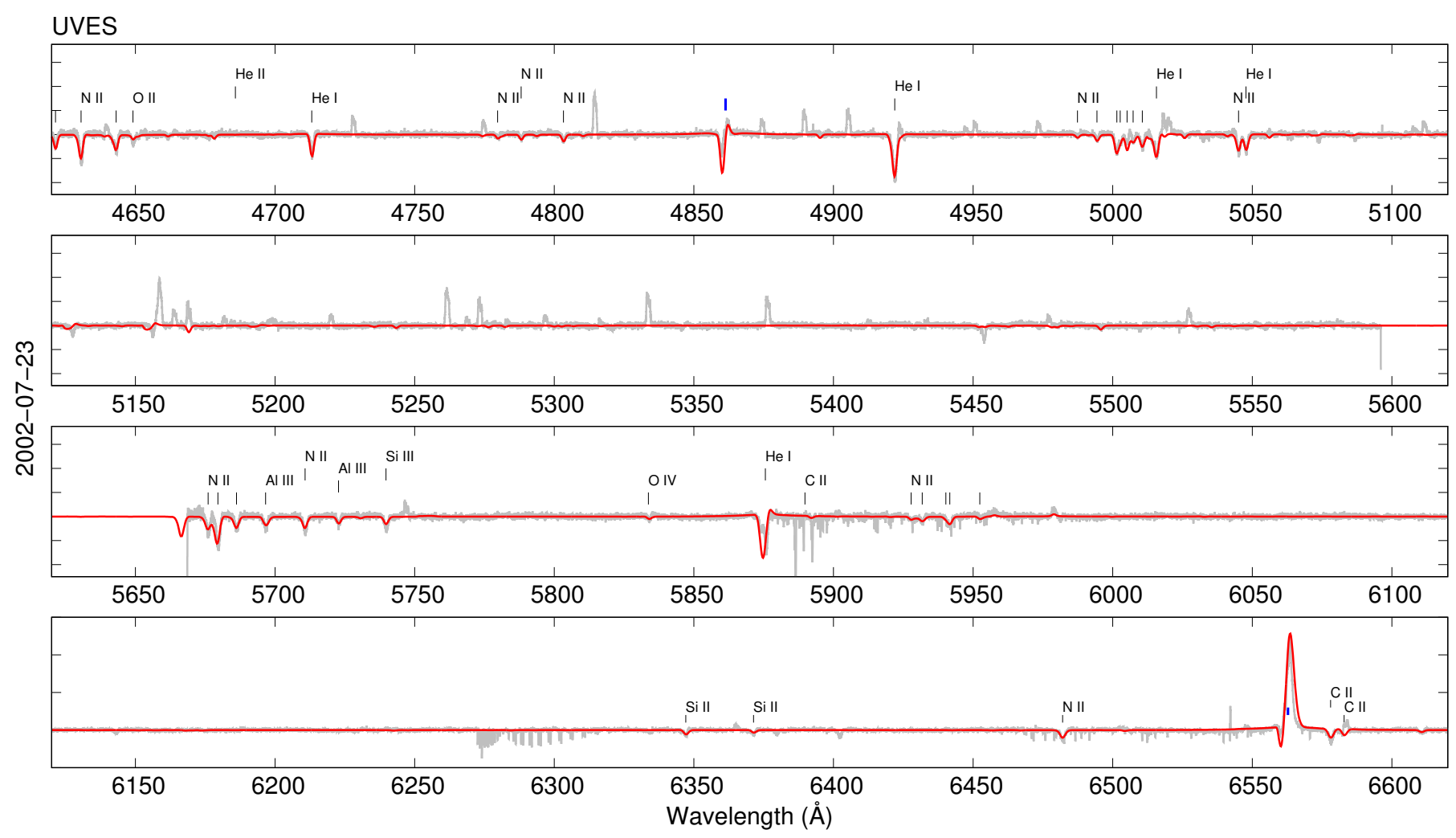

Fig. 10. Best-fit model via CMFGEN compared to the UVES spectrum obtained on 23 July 2002. The blue marks indicate hydrogen lines. The unfitted emission lines are nebular [Fe II] lines.

stellar parameters of R71. The modeling criteria are described in Sect. 2.4. Small spectral variations, such as the strength of hydrogen lines, can be observed in this data set. However, for simplicity and lack of simultaneous photometry for each spectral epoch, we determine the average of the fundamental stellar parameters over the time span of the quiescent state of the star. During the outburst, R71 has effective temperatures of 6000-6500 K and is too cool to be modeled with the (hot star wind) code CMFGEN.

Figure 10 shows the 2002 UVES spectrum compared to our best-fit CMFGEN model. The other spectral epochs and their comparison with the model are in Appendix A (Figs. A.1 to A.3). Derived stellar parameters for quiescent state of R71 are listed in Table 3. We find an effective temperature of $T_{\text {eff }}=15500 \pm$ $500 \mathrm{~K}$ and a stellar luminosity of $\log \left(L / L_{\odot}\right)=5.78 \pm 0.02$ $\left(M_{\text {bol }}=-9.65 \mathrm{mag}\right)$. We use the blue edge of the $\mathrm{H} \alpha$ PCyg absorption and other Balmer lines as a proxy for the wind terminal velocity. Since the absorption is not well defined and filled in with extra emission this measurement gives only a lower limit of $v_{\infty}=190 \mathrm{~km} \mathrm{~s}^{-1}$. From the metallic resonance line $\mathrm{Mg}$ II 2803, observed in ultraviolet IUE spectra obtained in 1981, 1985, and 1986, when the star was in a quiescent state, we determine a terminal velocity of $v_{\infty}=203 \pm 2 \mathrm{~km} \mathrm{~s}^{-1}$. An additional higher velocity component is observed, which extends to $v_{\infty}=281 \pm 7 \mathrm{~km} \mathrm{~s}^{-1}$. The gravity is estimated to be $\log (g /[\mathrm{cgs}])=1.80$ and the stellar mass to $27 M_{\odot}$. A mass-loss rate of $4.0 \times 10^{-6} M_{\odot} \mathrm{yr}^{-1}$ for a clumping factor equal to 1 fits the hydrogen lines well. However, extended emission from surrounding material is apparent in the spectra and not all hydrogen and helium lines are matched equally well.

\subsection{Surface chemical abundances}

We determine abundances of $\mathrm{H}, \mathrm{He}$, and the $\mathrm{CNO}$ elements as described in Sect. 2.4. Table 4 provides an overview of the
Table 3. Fundamental stellar parameters of R71 during the quiescent phase of the star (from our best-fit CMFGEN model).

\begin{tabular}{lcc}
\hline \hline Parameter & Value & Error \\
\hline$T_{\text {eff }}^{a}$ & $15500 \mathrm{~K}$ & $500 \mathrm{~K}$ \\
$T_{*}^{b}$ & $16200 \mathrm{~K}$ & \\
$\log \left(L_{*} / L_{\odot}\right)$ & 5.78 & 0.02 \\
$M_{\text {bol }}$ & -9.65 & 0.02 \\
$M$ & $4.0 \times 10^{-6} M_{\odot} \mathrm{yr}^{-1}$ & $0.5 \times 10^{-6} M_{\odot} \mathrm{yr}^{-1}$ \\
$\log (g /[\mathrm{cgs}])^{c}$ & 1.80 & 0.1 \\
$M$ & $27 M_{\odot}$ & \\
$R_{\text {phot }}$ & $107 R_{\odot}$ & \\
$R_{*}$ & $98 R_{\odot}$ & $5 \mathrm{~km} \mathrm{~s}^{-1}$ \\
$v_{\text {turb }}$ & $15 \mathrm{~km} \mathrm{~s}^{-1}$ & $10 \mathrm{~km} \mathrm{~s}^{-1}$ \\
$v_{\infty}$ & $190 \mathrm{~km} \mathrm{~s}^{-1}$ & $0.1 \mathrm{mag}^{-1}$ \\
$A_{V}$ & $0.6 \mathrm{mag}$ & \\
\hline
\end{tabular}

Notes. ${ }^{(a)}$ Parameters labeled "eff" and "phot" refer to $\tau_{\text {ROSS }}=2 / 3$. ${ }^{(b)}$ Parameters labeled "**" refer to $\tau_{\mathrm{ROSS}}=20 .{ }^{(c)}$ Specified at $\tau_{\mathrm{ROSS}}=$ $2 / 3$.

derived number fraction relative to hydrogen, the mass fraction, and the ratio to standard LMC abundances. The R71 abundances imply a strong enrichment of the atmosphere with $\mathrm{CNO}$ processed material.

We find similar CNO abundances as Lennon et al. (1993). These authors determined the abundances using CASPEC data, which only provide a very limited wavelength range from 37405445. In addition to the CASPEC spectra, we use UVES and FEROS spectra, covering the entire wavelength region from 3280-9200 ̊. Lennon et al. found number ratios of $\mathrm{He} / \mathrm{H}=$ $0.43, \mathrm{O} / \mathrm{N}=0.14$, and $\mathrm{C} / \mathrm{N}=0.03$, while we find number ratios of $\mathrm{He} / \mathrm{H}=0.20, \mathrm{O} / \mathrm{N}=0.08$, and $\mathrm{C} / \mathrm{N}=0.05$. Determining the 
Table 4. Surface chemical abundances for R71. Error estimates are on a $10-20 \%$ level. For He the error may be as large as $50 \%$.

\begin{tabular}{cccc}
\hline \hline Species & $\begin{array}{c}\text { Number fraction } \\
\text { (relative to H) }\end{array}$ & Mass fraction $^{a}$ & $Z / Z_{\mathrm{LMC}}$ \\
\hline $\mathrm{H}$ & 1.0 & $5.5 \mathrm{e}-01$ & 0.745 \\
$\mathrm{He}$ & 0.2 & $4.4 \mathrm{e}-01$ & 1.719 \\
$\mathrm{C}$ & $3.0 \mathrm{e}-05$ & $2.0 \mathrm{e}-04$ & 0.204 \\
$\mathrm{~N}$ & $6.0 \mathrm{e}-04$ & $4.6 \mathrm{e}-03$ & 16.292 \\
$\mathrm{O}$ & $5.0 \mathrm{e}-05$ & $4.4 \mathrm{e}-04$ & 0.180 \\
\hline
\end{tabular}

Notes. Derived from the 1984-2005 pre-outburst spectra. ${ }^{(a)}$ LMC mass fraction: $\mathrm{H}=7.38 \mathrm{e}-01, \mathrm{He}=2.56 \mathrm{e}-01, \mathrm{C}=9.79 \mathrm{e}-04, \mathrm{~N}=2.82 \mathrm{e}-04$, $\mathrm{O}=2.45 \mathrm{e}-03$.

He abundance is difficult and the error bars are large, i.e., we can fit the spectra using number ratios of $\mathrm{He} / \mathrm{H}$ between 0.10 and 0.3 . We find that a microturbulence of $10-20 \mathrm{~km} \mathrm{~s}^{-1}$ matches most lines, while the He I 6678, 7065 lines are best matched with $v_{\text {turb }} \approx 30 \mathrm{~km} \mathrm{~s}^{-1}$. These values correspond well to literature values of supergiants: Trundle et al. (2002) determined microturbulences for M 31 and Galactic supergiants in the range of $19 \mathrm{~km} \mathrm{~s}^{-1}$ to $35 \mathrm{~km} \mathrm{~s}^{-1}$ and Trundle et al. (2007) find microturbulences in Galactic, LMC, and SMC B-type stars between $0 \mathrm{~km} \mathrm{~s}^{-1}$ to $20 \mathrm{~km} \mathrm{~s}^{-1}$.

\section{Discussion}

\subsection{Evolutionary state}

To better understand the LBV instability phenomenon it is important to interpret the evolutionary state of LBVs and their evolutionary history. In the case of R71, it is crucial to know the luminosity because it may imply different evolutionary paths (see Sect. 1). However, there is ambiguity in the literature regarding the luminosity of R71 (Wolf et al. 1981; Lennon et al. 1993). The reason for this uncertainty lies in the different extinction values and effective temperatures (and thus bolometric corrections) assumed by different authors (Table 2 in Mehner et al. 2013). Support for the classification of R71 as a less luminous LBV came from the fact that its visual light and temperature during the 1970s outburst had much smaller variations than are observed for the more luminous LBVs (Thackeray 1974; Wolf et al. 1981). However, the current outburst displays every sign of an outburst of a classical LBV and it appears not to be uncommon that outbursts of the same star can have different magnitudes (Walborn et al. 2017). The large differences in S Doradus outbursts for the same star should be taken into account when discussing potential causes for the instability. It also renders the classification scheme based on outburst magnitude futile.

Figure 11 shows the location of R71 in the HR diagram during quiescence (determined in this paper) and during its current outburst state (determined in Mehner et al. 2013). The four open red squares indicate literature parameters (Wolf et al. 1981; van Genderen 1982; van Genderen et al. 1988; Lennon et al. 1993). Their substantial spread across the HR diagram demonstrates the large errors associated with stellar parameters published for LBVs - even when the distance is well known as in the case of R71. With our CMFGEN atmosphere modeling analysis we can finally settle the luminosity debate. R71 occupies the region of classical LBVs in the HR diagram, but at the lower luminosity end. A previous RSG phase, which in particular composition studies support (Voors et al. 1999; van Loon et al. 2010; Boyer et al. 2010), cannot be ruled out.
R71 has a strong surface enrichment in CNO processed material. It is, however, not possible to constrain its evolutionary state or deduce its evolutionary history based on it. Various (not well constrained) factors such as mass loss and mixing affect the evolution of the surface abundances strongly (Meynet et al. 2013) and the correct treatment of convection in stellar evolutionary codes is still uncertain (Georgy et al. 2014). Georgy et al. showed that the surface chemical abundances of blue supergiants are very sensitive to the way convection is modeled in the stellar evolution code (Ledoux criterion versus Schwarzschild criterion).

Smith \& Tombleson (2015) suggested that LBVs are the rejuvenated mass gainer products of massive star binary evolution, while Humphreys et al. (2016) argued that LBVs are predominantly the products of single star evolution. R71 is the most isolated LBV in the LMC from nearby O-type stars (Smith \& Tombleson 2015) and Lennon et al. (2017) computed its space velocity using the Gaia TGAS proper motion catalog. Both studies support the case that R71 cannot have evolved as a single star because it lies too far from massive (OB-type) star forming complexes to have arrived at its current position during a lifetime as a single star. In the case of a binary, mass transfer from this putative companion may have rejuvenated R71. Maybe its S Doradus outbursts and the oscillatory variability discussed in Sect. 3.1.3 could be triggered or modulated by this putative close companion.

\subsection{Instabilities in the atmosphere during the SDoradus outburst}

During quiescent state, R71 shows microvariations of $\Delta m_{\mathrm{V}} \sim$ 0.1 mag on timescales of 14-100 days attributed to large turbulent elements and dynamical instabilities in the photosphere or to pulsations (van Genderen et al. 1985, 1988, 1997; Lamers et al. 1998). In this paper, we report on larger amplitude and longer period oscillations in the light curve and spectrum of R71 during the current outburst. The spectral variations, i.e., the occurrence of double absorption lines, have a cycle length of about $850 \mathrm{~d}$. The visual light curve shows sinusoidal variations of $\sim 0.2 \mathrm{mag}$ with a period of about $425 \mathrm{~d}$, hinting at period doubling. Variations in the R71 light curve are also seen during the rise in brightness in 2007 and 2008, but with a shorter timescale. This is qualitatively in agreement with pulsation because the mean density of the star was higher then.

R71 falls in the LBV instability strip in the HR diagram during its quiescent phase, but does not cross any other known location of pulsating variables during its outburst phase; see Fig. 1 in Jeffery \& Saio (2016). However, the Cepheid instability strip may extend to higher luminosities beyond the RV Tau variables (meshed yellow ellipse in Fig. 11). The much expanded, cool envelope during the outburst now engulfs the Fe II/Fe III ionization zone, which could provide driving through the $\kappa$-mechanism.

RV Tau variables are pulsating variables (Percy 2007). These objects are post-asymptotic giant branch stars with masses close to solar mass and spectral types ranging from early $F$ to early $\mathrm{K}$. The reasons for their variability are still not entirely clear, but they are supposedly seated in the outer photospheric layers. They may be caused by multiple pulsation modes present in the star or nonlinear or chaotic behavior. Indeed, the photometric and spectroscopic variations of R71 during outburst are similar to what is observed in RV Tau variables. The light curves of RV Tau stars are modulated in an irregular way with period doubling owing to a resonance between the fundamental mode and first 


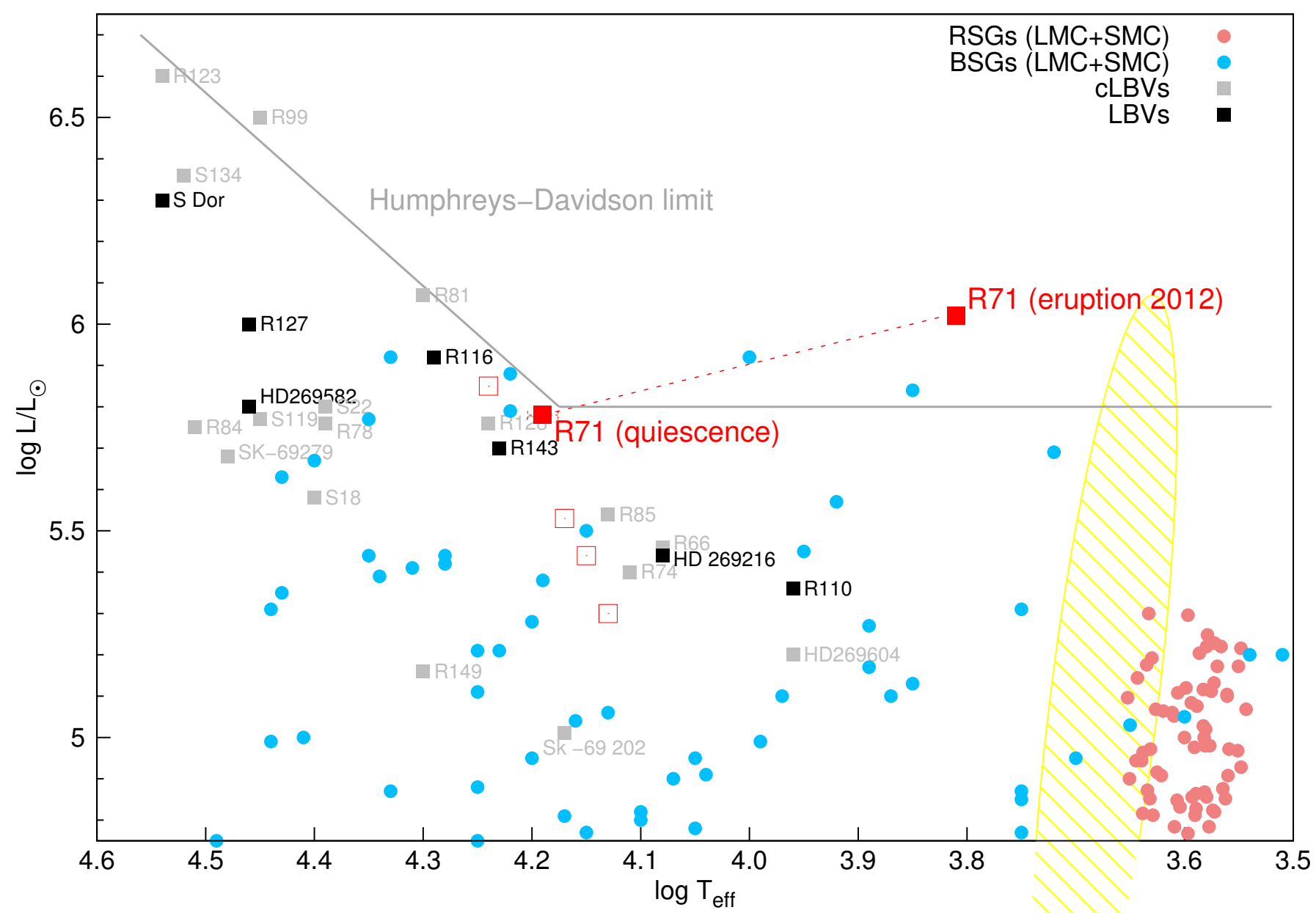

Fig. 11. Schematic upper HR diagram of the LMC. The solid gray curve is the upper luminosity boundary for the LMC (Humphreys \& Davidson 1979). Confirmed LBVs (black) and candidate LBVs (gray) are shown at their minimum phases (Zickgraf et al. 1986, 1996; van Genderen 2001; Tubbesing et al. 2002; Evans et al. 2004; Hainich et al. 2014). Blue and red supergiants are indicated with blue and red filled circles (Trundle et al. 2004; Trundle \& Lennon 2005; Levesque et al. 2006; Hunter et al. 2008). We show the locations of R71 during its quiescent state and its current outburst (filled red squares, parameters from this paper, and Mehner et al. 2013). Whether R71 really increased its bolometric luminosity during the outburst as indicated in this figure requires more detailed modeling beyond the scope of this paper. Previous literature values of the quiescent state of R71 are also shown (open red squares). The dashed yellow ellipse indicates the extension of the Cepheid instability strip.

overtone (e.g., Takeuti \& Petersen 1983; Moskalik \& Buchler 1990; Pollard et al. 1996), which may also be the case for R71. Also, double absorption lines have been observed in RV Tau stars, attributed to shock waves within the atmosphere (Gillet et al. 1989). Pulsations at such high stellar luminosities acting on a small amount of mass in the outer layers, are expected to be nonlinear, which accounts for the irregular behavior of the visual light curve.

While RV Tau variables cross the Cepheid instability during their evolution (Wallerstein 2002), S Doradus outbursts do not represent evolutionary transitions in the HR diagram. During outbursts, LBVs only appear to be relocated in the HR diagram, but their true evolutionary status does not change. The inner structure of an LBV in outburst might thus be far away from an evolutionary equilibrium and any relation to either Cepheid or RV Tau stars can only be based on phenomenology. It is also not clear if the phenomena observed for evolved lower mass stars can be extended to the atmospheres of LBVs during S Doradus outbursts. However, similar to RV Tau variables, LBVs in outburst have very extended, cool, low-mass outer atmospheres.

Maeder (1980) discusses supergiant pulsations with respect to their amplitude and period distribution across the HR diagram and their potential driving mechanisms. The author shows the importance of external convective zones present in these stars and discusses possibilities for non-radial oscillations resulting from either $g^{-}$modes in the presence of an extra-restoring force like rotation or the excitation of $g^{+}$modes. As stated by van Genderen et al. (1985), the microvariations of R71 during quiescent state fit well into the period-luminosity relation (Eq. (7) and Fig. 6 in Maeder 1980; a period of about $60 \mathrm{~d}$ is expected). Also, the observed oscillations during the current outburst with much larger amplitude and timescale fulfill well the period-luminosity relation; a period of $780 \mathrm{~d}$ is expected compared to the observed $850 \mathrm{~d}$. This suggests oscillations in the convective outer zones of R71. Other LBVs in outburst show similar oscillations in their light curve; see the case of R127 (Fig. 1 in Walborn et al. 2017). How the extended atmosphere of LBVs act in terms of convection and convective instabilities requires detailed theoretical attention. Under the conditions of extended LBV atmospheres, the classical $\kappa$ mechanism could be very efficient, i.e., minor changes in the temperature could cause large changes in opacity.

\section{Conclusions}

R71 was well-observed in recent decades covering its quiescent phases, its 1970-1977 S Doradus outburst, and its current outburst. The available multiwavelength data sets give us a unique 
opportunity to better understand the LBV phenomenon. The current S Doradus outburst of R71 has a much larger amplitude and longer duration than its previous outbursts in the 1970s and the first half of the 20th century. Consecutive outbursts, within decades, of the same star can be of rather different magnitude and a classification scheme of LBVs based on one outburst is thus insufficient.

We determined the fundamental stellar parameters of R71 using pre-outburst spectra and atmosphere models computed with CMFGEN. The effective temperature of the star is $T_{\text {eff }}=$ $15500 \pm 500 \mathrm{~K}$ and its stellar luminosity is $\log \left(L / L_{\odot}\right)=5.78 \pm$ 0.02 . R71 is thus at the lower luminosity end of the classical LBVs during quiescence. Other stellar wind parameters and abundances are provided in the paper. We also present the entire SED from the ultraviolet to the mid-infrared of the star in outburst. Mid-infrared observations suggest an increase of hot dust during the current outbursts and cooling of dust and grain evolution in the most outer regions.

The visual light curve and spectrum of R71 show oscillations during the current S Doradus outburst, resembling phenomena observed in the lower mass RV Tau stars. The occurrence of double absorption lines may result from shocks in the atmosphere and variations in the light curve may hint at period doubling. The timescale of these variations fit well into the period-luminosity relation for supergiants (Maeder 1980), which suggests oscillations in the convective outer zones of R71. Further analysis and theoretical modeling are required to investigate these oscillations and also to constrain the mechanisms of the LBV phenomenon and the evolutionary state of LBVs.

Acknowledgements. We acknowledge with thanks the variable star observation from the AAVSO International Database contributed by observers worldwide and used in this research https://www . aavso.org. ASAS data are available at http://www.astrouw.edu.pl/asas/. The IUE data used in this paper were obtained from the Mikulski Archive for Space Telescopes (MAST). STScI is operated by the Association of Universities for Research in Astronomy, Inc., under NASA contract NAS5-26555. Support for MAST for non-HST data is provided by the NASA Office of Space Science via grant NNX09AF08G and by other grants and contracts. This publication makes use of data products from the Wide-field Infrared Survey Explorer, which is a joint project of the University of California, Los Angeles and the Jet Propulsion Laboratory/California Institute of Technology, funded by the National Aeronautics and Space Administration. This research has made use of the NASA/IPAC Infrared Science Archive, which is operated by the Jet Propulsion Laboratory, California Institute of Technology, under contract with the National Aeronautics and Space Administration. This research has made use of NASA's Astrophysics Data System. CMFGEN can be downloaded at http://kookaburra.phyast.pitt.edu/hillier/web/CMFGEN . htm. This research made use of Astropy, a community-developed core Python package for Astronomy (Astropy Collaboration et al. 2013). C.A. acknowledges support from FONDECYT grant No. 3150463 and from the Ministry of Economy, Development, and Tourism's Millennium Science Initiative through grant IC120009, awarded to The Millennium Institute of Astrophysics, MAS.

\section{References}

Agliozzo, C., Umana, G., Trigilio, C., et al. 2012, MNRAS, 426, 181

Asmus, D., Hönig, S. F., Gandhi, P., Smette, A., \& Duschl, W. J. 2014, MNRAS, 439, 1648

Asplund, M., Grevesse, N., Sauval, A. J., \& Scott, P. 2009, ARA\&A, 47, 481

Astropy Collaboration, Robitaille, T. P., Tollerud, E. J., et al. 2013, A\&A, 558, A33

Barkat, Z., \& Wheeler, J. C. 1989, ApJ, 341, 925

Beltrán, M. T., \& de Wit, W. J. 2016, A\&ARv, 24, 6

Boffin, H. M. J., Rivinius, T., Mérand, A., et al. 2016, A\&A, 593, A90

Boyer, M. L., Sargent, B., van Loon, J. T., et al. 2010, A\&A, 518, L142

Chita, S. M., Langer, N., van Marle, A. J., García-Segura, G., \& Heger, A. 2008, A\&A, 488, L37

Clark, J. S., Crowther, P. A., Larionov, V. M., et al. 2009, A\&A, 507, 1555

Clark, J. S., Castro, N., Garcia, M., et al. 2012, A\&A, 541, A146

Cohen, M., Walker, R. G., Carter, B., et al. 1999, AJ, 117, 1864
Conti, P. S. 1984, in Observational Tests of the Stellar Evolution Theory, eds. A. Maeder, \& A. Renzini, IAU Symp., 105, 233

Conti, P. S. 1997, in Luminous Blue Variables: Massive Stars in Transition, eds. A. Nota, \& H. Lamers, PASPC, 120, 387

Cutri, R. M., Skrutskie, M. F., van Dyk, S., et al. 2003, VizieR Online Data Catalog: II/246

Cutri, R. M., Wright, E. L., Conrow, T., et al. 2013, VizieR Online Data Catalog: II $/ 328$

Davidson, K., \& Humphreys, R. M. 1997, ARA\&A, 35, 1

Davidson, K., \& Humphreys, R. M. 2012, Eta Carinae and the Supernova Impostors, Astrophys. Space Sci. Lib., 384

de Groot, M. 1988, Ir. Astron. J., 18, 163

Dekker, H., Delabre, B., \& Dodorico, S. 1986, in Instrumentation in Astronomy VI, ed. D. L. Crawford, Proc. SPIE, 627, 339

Dekker, H., D’Odorico, S., Kaufer, A., Delabre, B., \& Kotzlowski, H. 2000, in Optical and IR Telescope Instrumentation and Detectors, eds. M. Iye, \& A. F. Moorwood, Proc. SPIE, 4008, 534

Ducati, J. R. 2002, VizieR Online Data Catalog: II/237

Evans, C. J., Crowther, P. A., Fullerton, A. W., \& Hillier, D. J. 2004, ApJ, 610, 1021

Fitzpatrick, E. L. 1986, AJ, 92, 1068

Fitzpatrick, E. L. 1999, PASP, 111, 63

Fullerton, A. W., Massa, D. L., \& Prinja, R. K. 2006, ApJ, 637, 1025

Gal-Yam, A., \& Leonard, D. C. 2009, Nature, 458, 865

Gal-Yam, A., Leonard, D. C., Fox, D. B., et al. 2007, ApJ, 656, 372

Gamen, R., Barba, R., Walborn, N., et al. 2009, IAU Circ., 9082, 1

Gamen, R., Walborn, N., Morrell, N., Barba, R., \& Fernandez Lajus, E. 2012, Central Bureau Electronic Telegrams, 3192, 1

Georgiev, L., Koenigsberger, G., Hillier, D. J., et al. 2011, AJ, 142, 191

Georgy, C., Saio, H., \& Meynet, G. 2014, MNRAS, 439, L6

Gillet, D., Duquennoy, A., Bouchet, P., \& Gouiffes, C. 1989, A\&A, 215, 316

Glass, I. S. 1984, MNRAS, 209, 759

Gordon, K. D., Clayton, G. C., Misselt, K. A., Landolt, A. U., \& Wolff, M. J. 2003, ApJ, 594, 279

Gräfener, G., Owocki, S. P., \& Vink, J. S. 2012, A\&A, 538, A40

Grindlay, J., Tang, S., Los, E., \& Servillat, M. 2012, in New Horizons in Time Domain Astronomy, eds. E. Griffin, R. Hanisch, \& R. Seaman, IAU Symp., 285,29

Groh, J. H. 2011, J. Phys. Conf. Ser., 328, 012020

Groh, J. H., \& Vink, J. S. 2011, A\&A, 531, L10

Groh, J. H., Hillier, D. J., \& Damineli, A. 2006, ApJ, 638, L33

Groh, J. H., Hillier, D. J., Damineli, A., et al. 2009, ApJ, 698, 1698

Groh, J. H., Hillier, D. J., \& Damineli, A. 2011, ApJ, 736, 46

Groh, J. H., Meynet, G., \& Ekström, S. 2013, A\&A, 550, L7

Groh, J. H., Meynet, G., Ekström, S., \& Georgy, C. 2014, A\&A, 564, A30

Guha Niyogi, S., Min, M., Meixner, M., et al. 2014, A\&A, 569, A80

Hainich, R., Rühling, U., Todt, H., et al. 2014, A\&A, 565, A27

Hambsch, F.-J. 2012, Journal of the American Association of Variable Star Observers (JAAVSO), 40, 1003

Hillier, D. J. 1991, A\&A, 247, 455

Hillier, D. J., \& Allen, D. A. 1992, A\&A, 262, 153

Hillier, D. J., \& Miller, D. L. 1998, ApJ, 496, 407

Hillier, D. J., Davidson, K., Ishibashi, K., \& Gull, T. 2001, ApJ, 553, 837

Högbom, J. A. 1974, A\&AS, 15, 417

Houck, J. R., Roellig, T. L., van Cleve, J., et al. 2004, ApJS, 154, 18

Humphreys, R. M., \& Davidson, K. 1979, ApJ, 232, 409

Humphreys, R. M., \& Davidson, K. 1984, Science, 223, 243

Humphreys, R. M., \& Davidson, K. 1994, PASP, 106, 1025

Humphreys, R. M., Weis, K., Davidson, K., \& Gordon, M. S. 2016, ApJ, 825, 64

Hunter, I., Lennon, D. J., Dufton, P. L., et al. 2008, A\&A, 479, 541

Jeffery, C. S., \& Saio, H. 2016, MNRAS, 458, 1352

Justham, S., Podsiadlowski, P., \& Vink, J. S. 2014, ApJ, 796, 121

Kaufer, A., Stahl, O., Tubbesing, S., et al. 1999, The Messenger, 95, 8

Käufl, H. U., Kerber, F., Asmus, D., et al. 2015, The Messenger, 159, 15

Kerber, F., Käufl, H. U., Tristram, K., et al. 2016, in Ground-based and Airborne Instrumentation for Astronomy VI, Proc. SPIE, 9908, 99080D

Kessler-Silacci, J., Augereau, J.-C., Dullemond, C. P., et al. 2006, ApJ, 639, 275

Lagage, P. O., Pel, J. W., Authier, M., et al. 2004, The Messenger, 117, 12

Lamers, H. J. G. L. M., \& de Groot, M. J. H. 1992, A\&A, 257, 153

Lamers, H. J. G. L. M., Bastiaanse, M. V., Aerts, C., \& Spoon, H. W. W. 1998, A\&A, 335, 605

Langer, N., Hamann, W.-R., Lennon, M., et al. 1994, A\&A, 290, 819

Le Luyer, M., Melnick, J., \& Richter, W. 1979, The Messenger, 17, 27

Lennon, D. J., Wobig, D., Kudritzki, R.-P., \& Stahl, O. 1993, Space Sci. Rev., 66, 207

Lennon, D. J., van der Marel, R. P., Ramos Lerate, M., O’Mullane, W., \& Sahlmann, J. 2017, A\&A, 603, A75

Levesque, E. M., Massey, P., Olsen, K. A. G., et al. 2006, ApJ, 645, 1102 
Lomb, N. R. 1976, Ap\&SS, 39, 447

Macchetto, F. 1976, Mem. Soc. Astron. It., 47, 431

Maeder, A. 1980, A\&A, 90, 311

Maeder, A. 1983, A\&A, 120, 113

Martins, F. 2011, Bulletin de la Société Royale des Sciences de Liège, 80, 29

McMullin, J. P., Waters, B., Schiebel, D., Young, W., \& Golap, K. 2007, in Astronomical Data Analysis Software and Systems XVI, eds. R. A. Shaw, F. Hill, \& D. J. Bell, ASP Conf. Ser., 376, 127

Mehner, A., Baade, D., Rivinius, T., et al. 2013, A\&A, 555, A116

Meynet, G., Ekstrom, S., Maeder, A., et al. 2013, in Lect. Notes Phys., eds M. Goupil, K. Belkacem, C. Neiner, F. Lignières, \& J. J. Green (Berlin: Springer Verlag), 865, 3

Mitchell, R. M., \& Robinson, G. 1986, MNRAS, 222, 347

Moskalik, P., \& Buchler, J. R. 1990, ApJ, 355, 590

Nota, A., \& Lamers, H. 1997, Luminous Blue Variables: Massive Stars in Transition, ASPC, 120

Ochsenbein, F., Bauer, P., \& Marcout, J. 2000, A\&AS, 143, 23

Panagia, N. 1973, AJ, 78, 929

Pecaut, M. J., \& Mamajek, E. E. 2013, ApJS, 208, 9

Percy, J. R. 2007, Understanding Variable Stars (Cambridge: Cambridge University Press)

Pietrzyński, G., Graczyk, D., Gieren, W., et al. 2013, Nature, 495, 76

Podsiadlowski, P. 1992, PASP, 104, 717

Pojmanski, G. 1997, Acta Astron., 47, 467

Pollard, K. R., Cottrell, P. L., Kilmartin, P. M., \& Gilmore, A. C. 1996, MNRAS, 279, 949

Puls, J., Vink, J. S., \& Najarro, F. 2008, A\&ARv, 16, 209

Quataert, E., \& Shiode, J. 2012, MNRAS, 423, L92

Sanyal, D., Grassitelli, L., Langer, N., \& Bestenlehner, J. M. 2015, A\&A, 580, A20

Sault, R. J., Teuben, P. J., \& Wright, M. C. H. 1995, in Astronomical Data Analysis Software and Systems IV, eds. R. A. Shaw, H. E. Payne, \& J. J. E. Hayes, ASP Conf. Ser., 77, 433

Scargle, J. D. 1982, ApJ, 263, 835

Smith, N. 2007, AJ, 133, 1034

Smith, N., \& Tombleson, R. 2015, MNRAS, 447, 598
Smith, N., Vink, J. S., \& de Koter, A. 2004, ApJ, 615, 475 Smith, N., Li, W., Foley, R. J., et al. 2007, ApJ, 666, 1116 Smith, N., Chornock, R., Li, W., et al. 2008, ApJ, 686, 467 Stahl, O., \& Wolf, B. 1986, A\&A, 158, 371

Sundqvist, J. O., \& Owocki, S. P. 2013, MNRAS, 428, 1837

Sundqvist, J. O., Puls, J., \& Owocki, S. P. 2014, A\&A, 568, A59

Takeuti, M., \& Petersen, J. O. 1983, A\&A, 117, 352

Thackeray, A. D. 1974, MNRAS, 168, 221

Trundle, C., \& Lennon, D. J. 2005, A\&A, 434, 677

Trundle, C., Dufton, P. L., Lennon, D. J., Smartt, S. J., \& Urbaneja, M. A. 2002, A\&A, 395, 519

Trundle, C., Lennon, D. J., Puls, J., \& Dufton, P. L. 2004, A\&A, 417, 217

Trundle, C., Dufton, P. L., Hunter, I., et al. 2007, A\&A, 471, 625

Tubbesing, S., Kaufer, A., Stahl, O., et al. 2002, A\&A, 389, 931

van Genderen, A. M. 1979, A\&AS, 38, 151

van Genderen, A. M. 1982, A\&A, 112, 61

van Genderen, A. M. 2001, A\&A, 366, 508

van Genderen, A. M., Steemers, W. J. G., Feldbrugge, P. T. M., et al. 1985, A\&A, 153,163

van Genderen, A. M., The, P. S., Augusteijn, T., et al. 1988, A\&AS, 74, 453

van Genderen, A. M., de Groot, M., \& Sterken, C. 1997, A\&AS, 124, 517

van Loon, J. T., Oliveira, J. M., Gordon, K. D., et al. 2010, AJ, 139, 68

Vernet, J., Dekker, H., D’Odorico, S., et al. 2011, A\&A, 536, A105

Voors, R. H. M., Waters, L. B. F. M., Morris, P. W., et al. 1999, A\&A, 341, L67

Walborn, N. R., Gamen, R. C., Morrell, N. I., et al. 2017, AJ, 154, 15

Wallerstein, G. 2002, PASP, 114, 689

Weis, K. 2003, A\&A, 408, 205

Wolf, B. 1975, A\&A, 41, 471

Wolf, B., \& Stahl, O. 1990, A\&A, 235, 340

Wolf, B., \& Zickgraf, F. J. 1986, A\&A, 164, 435

Wolf, B., Appenzeller, I., \& Stahl, O. 1981, A\&A, 103, 94

Yusof, N., Hirschi, R., Meynet, G., et al. 2013, MNRAS, 433, 1114

Zickgraf, F.-J., Wolf, B., Leitherer, C., Appenzeller, I., \& Stahl, O. 1986, A\&A, 163,119

Zickgraf, F.-J., Humphreys, R. M., Lamers, H. J. G. L. M., et al. 1996, A\&A, 315,510 


\section{Appendix A: CMFGEN models}

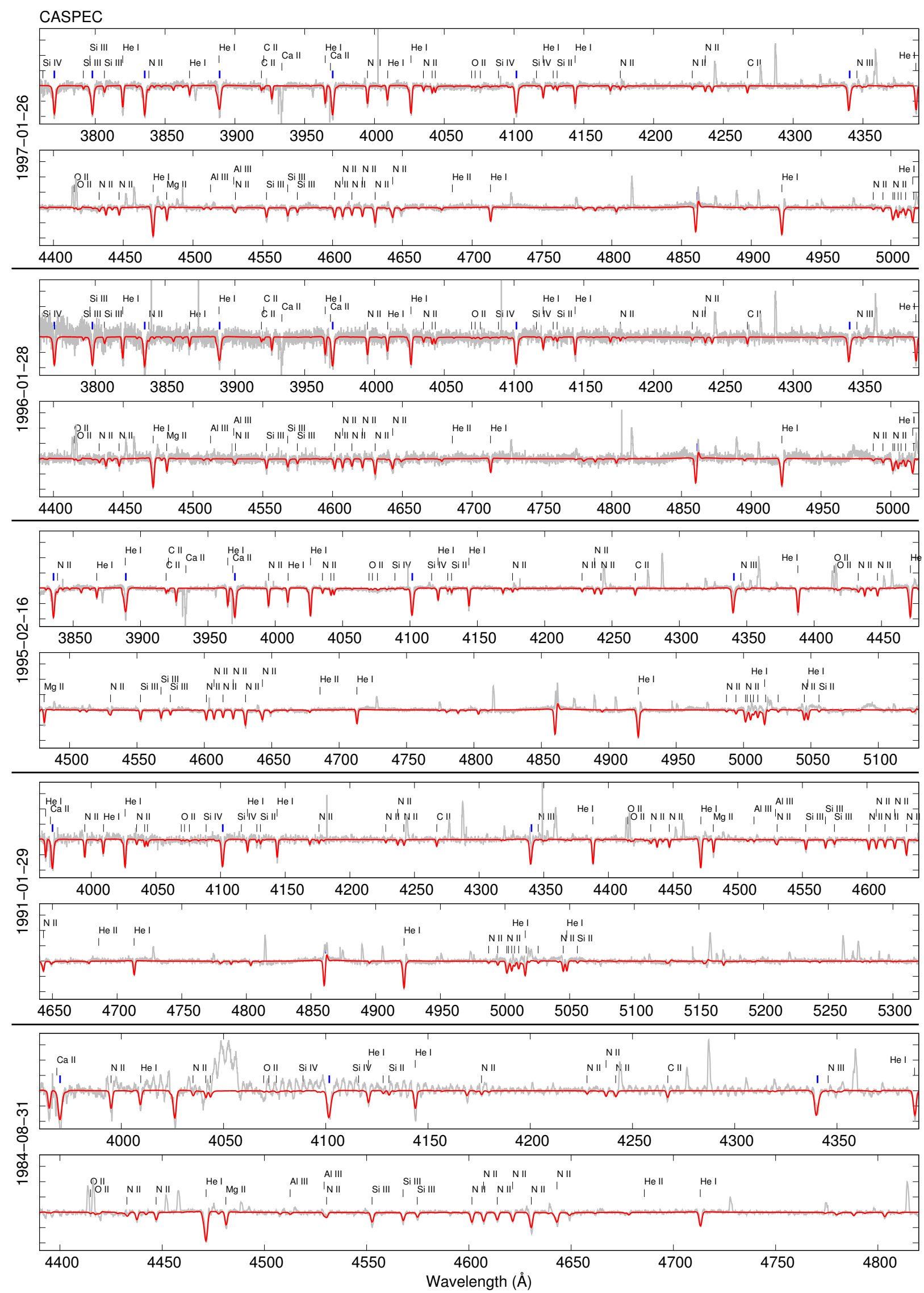

Fig. A.1. Best-fit model of CMFGEN compared to the CASPEC data from 1984 to 1997 . The many emission lines are nebular [Fe II] and [N II] lines. The blue marks indicate hydrogen lines. 


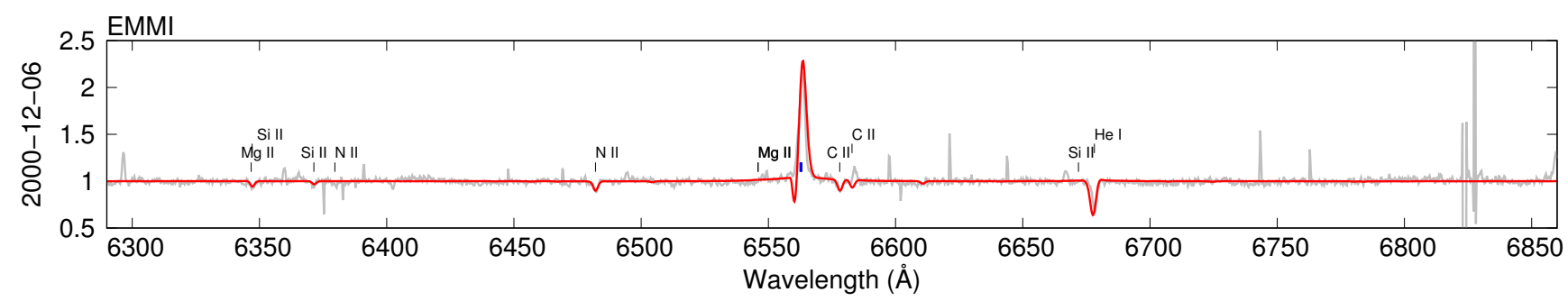

Fig. A.2. Best-fit model of CMFGEN compared to the EMMI data in 2000.

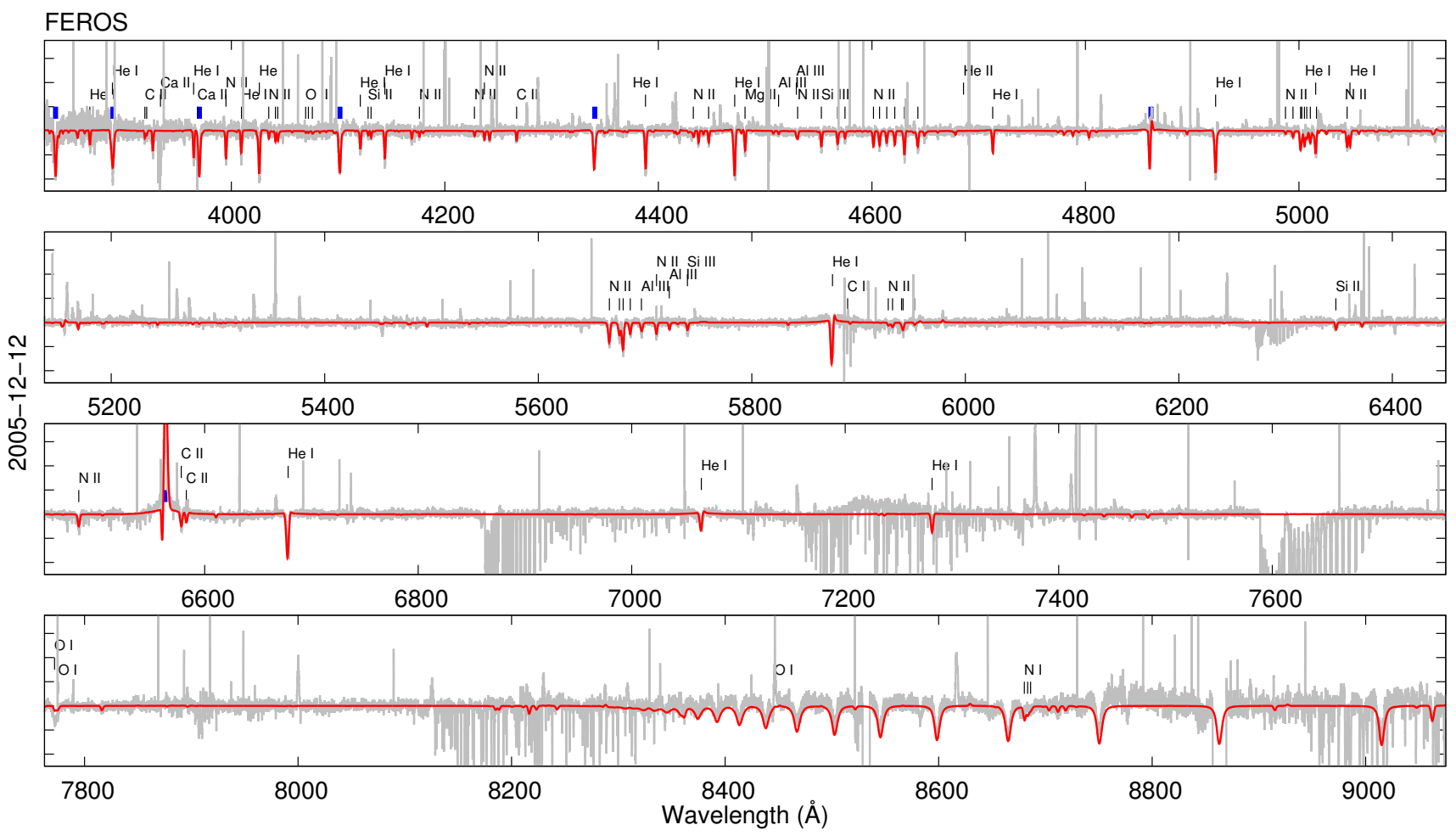

Fig. A.3. Atmosphere models of CMFGEN compared to the FEROS spectra obtained on 2005-12-12. 


\section{Appendix B: Journal of spectroscopic observations}

Table B.1. Journal of spectroscopic observations.

\begin{tabular}{|c|c|c|c|c|c|}
\hline Date & $\begin{array}{c}\text { MJD } \\
\text { (days) }\end{array}$ & Instrument & $\begin{array}{l}\text { Wavelength range } \\
(\AA)\end{array}$ & $\begin{array}{c}\text { Spectral resolving } \\
\text { power }\end{array}$ & $\begin{array}{l}\text { Total exposure time } \\
\text { (s) }\end{array}$ \\
\hline 1984-08-31 & 45943 & CASPEC & $3895-4970$ & 20000 & 1500 \\
\hline $1991-01-29$ & 48285 & CASPEC & $3920-5445$ & 20000 & 1200 \\
\hline $1995-02-16$ & 49764 & CASPEC & $3815-5140$ & 20000 & 3000 \\
\hline $1996-01-28$ & 50110 & CASPEC & $3740-5045$ & 20000 & 756 \\
\hline 1997-01-26 & 50474 & CASPEC & $3765-5045$ & 20000 & 1200 \\
\hline $2000-12-06$ & 51884.2 & EMMI & $6240-6870$ & 20000 & 600,1800 \\
\hline $2002-07-23$ & 52478.4 & UVES $^{1}$ & $3280-4560$ & 40000 & 360 \\
\hline $2002-07-23$ & 52478.4 & UVES & 4580-6690 & 40000 & $3 \times 110$ \\
\hline $2002-08-03$ & 52489.4 & UVES & $3280-4560$ & 40000 & 360 \\
\hline 2002-08-03 & 52489.4 & UVES & $4580-6690$ & 40000 & $3 \times 110$ \\
\hline 2002-08-12 & 52498.4 & UVES & $3280-4560$ & 40000 & 360 \\
\hline 2002-08-12 & 52498.4 & UVES & $4580-6690$ & 40000 & $3 \times 110$ \\
\hline 2002-09-04 & 52521.4 & UVES & $3280-4560$ & 40000 & 360 \\
\hline 2002-09-04 & 52521.4 & UVES & $4580-6690$ & 40000 & $3 \times 110$ \\
\hline 2002-09-10 & 52527.4 & UVES & $3280-4560$ & 40000 & 360 \\
\hline 2002-09-10 & 52527.4 & UVES & 4580-6690 & 40000 & $3 \times 110$ \\
\hline $2002-09-18$ & 52535.3 & UVES & $3280-4560$ & 40000 & 360 \\
\hline 2002-09-18 & 52535.3 & UVES & $4580-6690$ & 40000 & $3 \times 110$ \\
\hline $2002-09-22$ & 52539.3 & UVES & $3280-4560$ & 40000 & 360 \\
\hline 2002-09-22 & 52539.3 & UVES & $4580-6690$ & 40000 & $3 \times 110$ \\
\hline 2002-09-30 & 52547.4 & UVES & $3280-4560$ & 40000 & 360 \\
\hline 2002-09-30 & 52547.4 & UVES & $4580-6690$ & 40000 & $3 \times 110$ \\
\hline $2002-10-06$ & 52553.4 & UVES & $3280-4560$ & 40000 & 360 \\
\hline 2002-10-06 & 52553.4 & UVES & 4580-6690 & 40000 & $3 \times 110$ \\
\hline $2005-12-12$ & 53716.2 & FEROS & $3500-9200$ & 48000 & $2 \times 450$ \\
\hline $2007-02-22$ & 54153.2 & FEROS & $3500-9200$ & 48000 & $3 \times 500$ \\
\hline 2011-02-13 & 55605.0 & FEROS & $3500-9200$ & 48000 & 900 \\
\hline 2011-03-20 & 55641.0 & FEROS & $3500-9200$ & 48000 & 1800 \\
\hline $2011-05-15$ & 55697.0 & FEROS & $3500-9200$ & 48000 & 1800 \\
\hline 2012-06-19 & 56097.4 & FEROS & $3500-9200$ & 48000 & 1800 \\
\hline $2014-11-29$ & 56990.1 & FEROS & $3500-9200$ & 48000 & $2 \times 960$ \\
\hline $2015-10-16$ & 57311.3 & FEROS & $3500-9200$ & 48000 & $2 \times 500$ \\
\hline $2012-08-26$ & 56165.3 & X-shooter ${ }^{2}$ & $10240-24800$ & 10000 & $3 \times 60,2 \times 10$ \\
\hline $2012-08-26$ & 56165.3 & X-shooter & $3000-5595$ & 9000 & $3 \times 120,2 \times 20$ \\
\hline $2012-08-26$ & 56165.3 & X-shooter & $5595-10240$ & 17000 & $3 \times 120,2 \times 20$ \\
\hline 2012-09-04 & 56174.3 & X-shooter & $10240-24800$ & 10000 & $3 \times 60,2 \times 10$ \\
\hline 2012-09-04 & 56174.3 & X-shooter & $3000-5595$ & 9000 & $3 \times 120,2 \times 20$ \\
\hline 2012-09-04 & 56174.3 & X-shooter & $5595-10240$ & 17000 & $3 \times 120,2 \times 20$ \\
\hline $2012-11-01$ & 56232.2 & X-shooter & $10240-24800$ & 10000 & $3 \times 60,2 \times 10$ \\
\hline 2012-11-01 & 56232.2 & X-shooter & $3000-5595$ & 9000 & $3 \times 120,2 \times 20$ \\
\hline 2012-11-01 & 56232.2 & X-shooter & $5595-10240$ & 17000 & $3 \times 120,2 \times 20$ \\
\hline 2013-02-02 & 56325.0 & X-shooter & $10240-24800$ & 10000 & $3 \times 60,2 \times 10$ \\
\hline 2013-02-02 & 56325.0 & X-shooter & $3000-5595$ & 9000 & $3 \times 120,2 \times 20$ \\
\hline $2013-02-02$ & 56325.0 & X-shooter & $5595-10240$ & 17000 & $3 \times 120,2 \times 20$ \\
\hline 2013-03-01 & 56352.1 & X-shooter & $10240-24800$ & 10000 & $3 \times 60,2 \times 10$ \\
\hline 2013-03-01 & 56352.1 & X-shooter & $3000-5595$ & 9000 & $3 \times 120,2 \times 20$ \\
\hline 2013-03-01 & 56352.1 & X-shooter & $5595-10240$ & 17000 & $3 \times 120,2 \times 20$ \\
\hline $2013-08-04$ & 56508.4 & X-shooter & $10240-24800$ & 10000 & $4 \times 60,2 \times 10$ \\
\hline 2013-08-04 & 56508.4 & X-shooter & $3000-5595$ & 9000 & $4 \times 120,2 \times 20$ \\
\hline $2013-08-04$ & 56508.4 & X-shooter & $5595-10240$ & 17000 & $4 \times 120,2 \times 20$ \\
\hline 2013-09-19 & 56554.3 & X-shooter & $10240-24800$ & 10000 & $4 \times 60,2 \times 10$ \\
\hline 2013-09-19 & 56554.3 & X-shooter & $3000-5595$ & 9000 & $4 \times 120,2 \times 20$ \\
\hline 2013-09-19 & 56554.3 & X-shooter & $5595-10240$ & 17000 & $4 \times 120,2 \times 20$ \\
\hline $2013-10-24$ & 56589.3 & X-shooter & $10240-24800$ & 10000 & $3 \times 60,2 \times 10$ \\
\hline
\end{tabular}

Notes. ${ }^{(1)}$ UVES slit width: $1^{\prime \prime} .{ }^{(2)} \mathrm{X}$-shooter slit width: 0 "'5/0"4/0".4 for the UVB/VIS/NIR arms and long exposures, $5^{\prime \prime} / 5^{\prime \prime} / 5^{\prime \prime}$ for the short exposures. 
Table B.1. continued.

\begin{tabular}{|c|c|c|c|c|c|}
\hline Date & $\begin{array}{l}\text { MJD } \\
\text { (days) }\end{array}$ & Instrument & $\begin{array}{l}\text { Wavelength range } \\
(\AA)\end{array}$ & $\begin{array}{c}\text { Spectral resolving } \\
\text { power }\end{array}$ & $\begin{array}{l}\text { Total exposure time } \\
\text { (s) }\end{array}$ \\
\hline 2013-10-24 & 56589.3 & X-shooter & $3000-5595$ & 9000 & $3 \times 120,2 \times 20$ \\
\hline 2013-10-24 & 56589.3 & X-shooter & $5595-10240$ & 17000 & $3 \times 120,2 \times 20$ \\
\hline 2013-11-12 & 56608.1 & X-shooter & $10240-24800$ & 10000 & $3 \times 60,2 \times 10$ \\
\hline $2013-11-12$ & 56608.1 & X-shooter & $3000-5595$ & 9000 & $3 \times 120,2 \times 20$ \\
\hline $2013-11-12$ & 56608.1 & X-shooter & $5595-10240$ & 17000 & $3 \times 120,2 \times 20$ \\
\hline $2013-12-22$ & 56648.3 & X-shooter & $10240-24800$ & 10000 & $3 \times 60,2 \times 10$ \\
\hline $2013-12-22$ & 56648.3 & X-shooter & $3000-5595$ & 9000 & $3 \times 120,2 \times 20$ \\
\hline $2013-12-22$ & 56648.3 & X-shooter & $5595-10240$ & 17000 & $3 \times 120,2 \times 20$ \\
\hline 2014-02-01 & 56689.2 & X-shooter & $10240-24800$ & 10000 & $3 \times 60,2 \times 10$ \\
\hline 2014-02-01 & 56689.2 & X-shooter & $3000-5595$ & 9000 & $3 \times 120,2 \times 20$ \\
\hline 2014-02-01 & 56689.2 & X-shooter & $5595-10240$ & 17000 & $3 \times 120,2 \times 20$ \\
\hline 2014-11-16 & 56977.1 & X-shooter & $10240-24800$ & 10000 & $3 \times 60,2 \times 10$ \\
\hline $2014-11-16$ & 56977.1 & X-shooter & $3000-5595$ & 9000 & $3 \times 120,2 \times 20$ \\
\hline $2014-11-16$ & 56977.1 & X-shooter & $5595-10240$ & 17000 & $3 \times 120,2 \times 20$ \\
\hline $2014-12-27$ & 57018.0 & X-shooter & $10240-24800$ & 10000 & $3 \times 60,2 \times 10$ \\
\hline $2014-12-27$ & 57018.0 & X-shooter & $3000-5595$ & 9000 & $3 \times 120,2 \times 20$ \\
\hline 2014-12-27 & 57018.0 & X-shooter & $5595-10240$ & 17000 & $3 \times 120,2 \times 20$ \\
\hline 2015-03-02 & 57083.1 & X-shooter & $10240-24800$ & 10000 & $3 \times 60,2 \times 10$ \\
\hline 2015-03-02 & 57083.1 & X-shooter & $3000-5595$ & 9000 & $3 \times 120,2 \times 20$ \\
\hline 2015-03-02 & 57083.1 & X-shooter & 5595-10240 & 17000 & $3 \times 120,2 \times 20$ \\
\hline $2015-10-15$ & 57310.3 & X-shooter & $10240-24800$ & 10000 & $3 \times 60,2 \times 10$ \\
\hline $2015-10-15$ & 57310.3 & X-shooter & $3000-5595$ & 9000 & $3 \times 120,2 \times 20$ \\
\hline 2015-10-15 & 57310.3 & X-shooter & $5595-10240$ & 17000 & $3 \times 120,2 \times 20$ \\
\hline $2015-12-18$ & 57374.3 & X-shooter & $10240-24800$ & 10000 & $3 \times 60,2 \times 10$ \\
\hline $2015-12-18$ & 57374.3 & X-shooter & 3000-5595 & 9000 & $3 \times 120,2 \times 20$ \\
\hline $2015-12-18$ & 57374.3 & X-shooter & $5595-10240$ & 17000 & $3 \times 120,2 \times 20$ \\
\hline 2016-02-19 & 57437.1 & X-shooter & $10240-24800$ & 10000 & $3 \times 60,2 \times 10$ \\
\hline 2016-02-19 & 57437.1 & X-shooter & $3000-5595$ & 9000 & $3 \times 120,2 \times 20$ \\
\hline 2016-02-19 & 57437.1 & X-shooter & 5 595-10 240 & 17000 & $3 \times 120,2 \times 20$ \\
\hline 2016-04-06 & 57484.0 & X-shooter & $10240-24800$ & 10000 & $4 \times 60,2 \times 10$ \\
\hline 2016-04-06 & 57484.0 & X-shooter & $3000-5595$ & 9000 & $4 \times 120,2 \times 20$ \\
\hline 2016-04-06 & 57484.0 & X-shooter & 5595-10240 & 17000 & $4 \times 120,2 \times 20$ \\
\hline 2016-08-03 & 57603.4 & X-shooter & $10240-24800$ & 10000 & $4 \times 60,2 \times 10$ \\
\hline 2016-08-03 & 57603.4 & X-shooter & 3000-5595 & 9000 & $4 \times 120,2 \times 20$ \\
\hline 2016-08-03 & 57603.4 & X-shooter & $5595-10240$ & 17000 & $4 \times 120,2 \times 20$ \\
\hline 2016-10-06 & 57667.4 & X-shooter & $10240-24800$ & 10000 & $4 \times 60,2 \times 10$ \\
\hline 2016-10-06 & 57667.4 & X-shooter & $3000-5595$ & 9000 & $4 \times 120,2 \times 20$ \\
\hline 2016-10-06 & 57667.4 & X-shooter & $5595-10240$ & 17000 & $4 \times 120,2 \times 20$ \\
\hline 2017-01-02 & 57755.3 & X-shooter & $10240-24800$ & 10000 & $4 \times 60,2 \times 10$ \\
\hline 2017-01-02 & 57755.3 & X-shooter & $3000-5595$ & 9000 & $4 \times 120,2 \times 20$ \\
\hline 2017-01-02 & 57755.3 & X-shooter & $5595-10240$ & 17000 & $4 \times 120,2 \times 20$ \\
\hline 2017-04-04 & 57847.0 & X-shooter & $10240-24800$ & 10000 & $4 \times 60,2 \times 10$ \\
\hline 2017-04-04 & 57847.0 & X-shooter & $3000-5595$ & 9000 & $4 \times 120,2 \times 20$ \\
\hline 2017-04-04 & 57847.0 & X-shooter & $5595-10240$ & 17000 & $4 \times 120,2 \times 20$ \\
\hline $2017-08-03$ & 57968.3 & X-shooter & $10240-24800$ & 10000 & $8 \times 60,4 \times 10$ \\
\hline 2017-08-03 & 57968.3 & X-shooter & $3000-5595$ & 9000 & $8 \times 120,4 \times 20$ \\
\hline 2017-08-03 & 57968.3 & X-shooter & $5595-10240$ & 17000 & $8 \times 120,4 \times 20$ \\
\hline
\end{tabular}

